\title{
Business Analytics and IT in Smart Grid - Part 1: The Impact of Measurement Differences on the iSHM Class Map Footprints of Overhead Low-Voltage Broadband over Power Lines Topologies
}

\author{
Athanasios G. Lazaropoulos ${ }^{1,2, *}$ \\ 1: School of Electrical and Computer Engineering / National Technical University of Athens / \\ 9 Iroon Polytechniou Street / Zografou, GR 15780 \\ 2: Department of Industrial Design and Production Engineering / School of Engineering / University of \\ West Attica / 250 Thivon \& P. Ralli / Athens, GR 12244
}

Received March 25, 2020; Accepted May 4, 2020; Published May 10, 2020

\begin{abstract}
Due to the smart grid (SG) operation, the power utilities are dealing with a cataclysm of big data that demands advanced information technology (IT) infrastructure and business analytics while one cause of this growth is the nature of the power grid operation that demands real-time measurements. In [1], [2], the theoretical framework and the numerical results for the interoperability of Deterministic Hybrid Model (DHM), initial Statistical Hybrid Model (iSHM), the definition procedure and the class maps have been presented for the overhead low-voltage broadband over power lines (OV LV BPL) networks as well as the iSHM footprints. On the basis of the five real indicative OV LV BPL topologies of [1], [2], the impact of measurement differences that follow either continuous uniform distributions (CUDs) or normal distributions (NDs) of different intensities is first highlighted on iSHM footprints.
\end{abstract}

Keywords: Smart Grid; Broadband over Power Lines (BPL) networks; Power Line Communications (PLC); Distribution and Transmission Power Grids; Capacity, Statistics; Business Analytics; IT; Modeling 


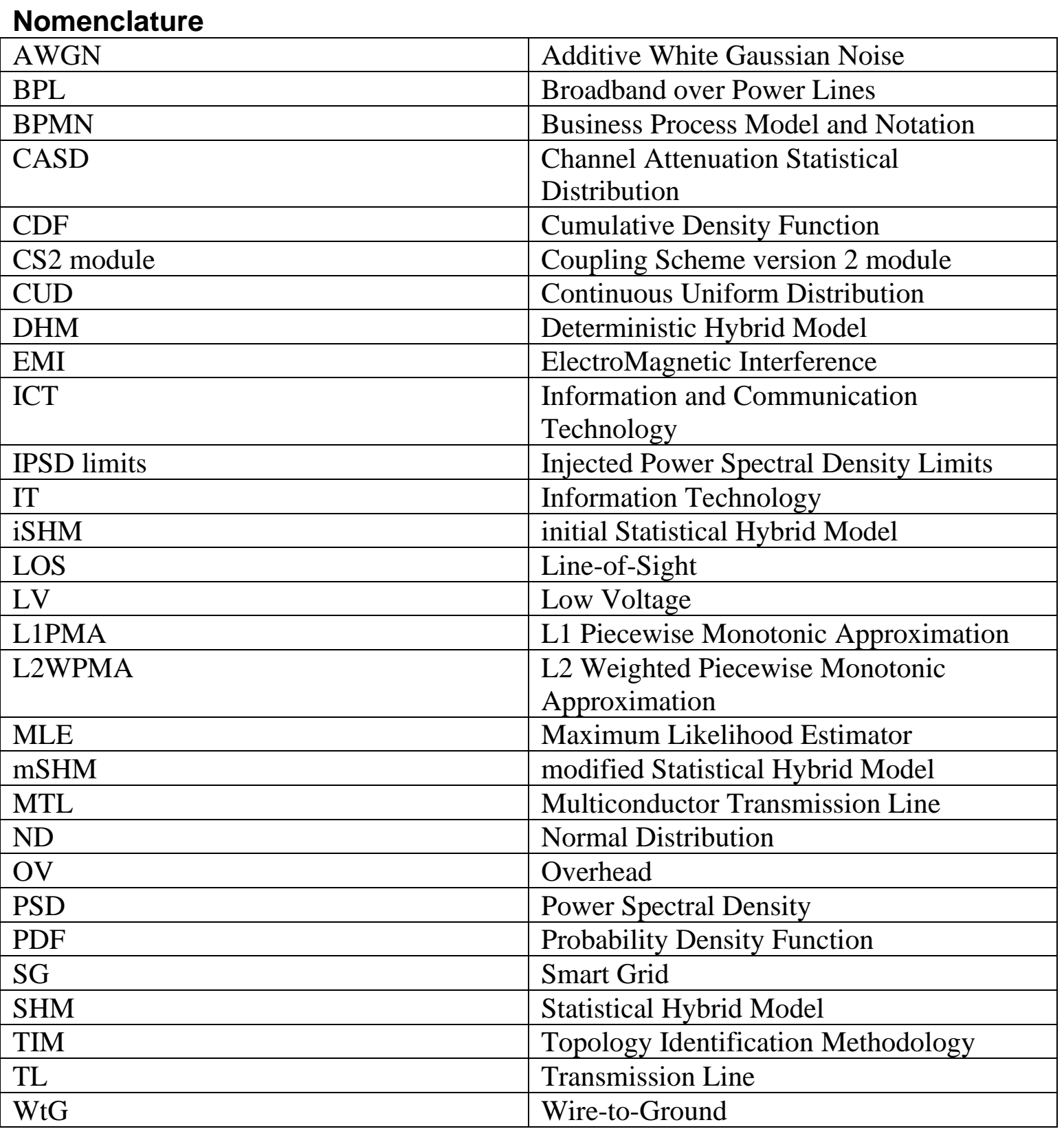




\section{Introduction}

To support various SG applications, the vintage transmission and distribution power grids need to obtain additional intelligence by rendering ITs as their key aspect [1]-[3]. Actually, the supported SG applications impose a big data challenge that requires IT infrastructure and advanced business analytics techniques in order to face with the vast amounts of data and their analytics [4], [5]. The adoption of the available SG ICT solutions may help towards the support of reliable and secured bi-directional data communications across the entire power grid, better power grid monitoring and management, further data integration of other power grid related sources, the improvement of power grid efficiency and the deal with fervent issues of modern energy networks [6]. Among the available SG ICT solutions, BPL networks attract the great attention from the stakeholders among the other available communications technologies since these networks can exploit the already installed wired power grid infrastructure without the need for additional wiring costs except for the ones that are related with network equipment [7]-[9].

As the operation of BPL networks is concerned across the power grid, a lot of evolution steps took place recently by focusing on the statistical processing of the communications channel that is proven to be a hostile medium for communications as the infrastructure and the equipment of transmission and distribution power grids are designed to deliver power rather information [1], [10]-[14]. On the basis of the well-validated DHM for transmission and distribution power grids [10], [11], [15]-[18], the proposed SHM framework, which consists of its iSHM and mSHM versions, has recently been proposed in [19]-[21]. Also, new tools that are integrated with SHM and further exploit its operation are available in [1], [2], [22]-[24], namely: (i) The definition procedure: This procedure enriches the existing BPL topology classes with virtual BPL topology subclasses statistically defined in terms of the applied SHM version and its corresponding successful CASD parameter pairs (i.e, MLEs and CDF for iSHM and mSHM CASDs, respectively); (ii) The class maps: 2D contour plots illustrate the borders between adjacent BPL topology classes while CASD parameter pairs with the corresponding BPL topology subclass average capacities are represented on the class map; and (iii) The class map footprints of critical events of the operation of power grids: The real OV LV BPL topologies, the real OV LV BPL topologies with a sole branch line fault and the real OV LV BPL topologies with a single hook for energy theft can be illustrated as superimposed white areas upon the class maps for given power grid type, SHM version, CASD, coupling scheme, IPSD limits and noise levels. In accordance with [2], the most descriptive class map footprints are the iSHM ones, which are going to be exploited in this paper, since their representation depends on a straightforward procedure rather than the approximation of mSHM ones. Until now, the results of iSHM and its accompanying tools have exploited as inputs the theoretical numerical results came from the operation of DHM.

Already been mentioned in [25]-[29], measurement differences between the experimental and theoretical results may occur due to a number of practical reasons and "real-life" difficulties thus having as a result the influence of the DHM numerical results in terms of the transfer function and capacity and, from now on, the destabilization of the entire iSHM framework operation (i.e., iSHM results as well as the accompanying tools). In accordance with [25], [26], [29], [30], a typical scenario to take into account 
measurement differences during the BPL network analysis is their handling as error distributions such as CUDs and NDs that are superimposed to the coupling scheme transfer function theoretical numerical results of DHM. In this paper, the impact of measurement differences as CUDs and NDs on the iSHM framework operation is first assessed through the iSHM class map footprints of a list of real indicative OV LV BPL topologies.

The rest of this paper is organized as follows: In Section II, the interaction among DHM, iSHM, the definition procedure, the class maps and footprints of OV LV BPL topologies is briefly outlined. Section III deals with the measurement differences and the mathematics concerning the integration of the corresponding measurement difference CUDs and NDs in SHM and its accompanying tools. In Section IV, the numerical results regarding the impact of measurement differences on the iSHM footprints of the aforementioned real indicative OV LV BPL topologies are shown. Section V concludes this paper.

\section{The Basics of DHM, iSHM, the Definition Procedure, the Class Maps and the Footprints of OV LV BPL Topologies}

Prior to examine the interaction of DHM, iSHM, the definition procedure, the class maps and the footprints of OV LV BPL topologies, brief details concerning the applied OV LV MTL configuration and indicative OV LV BPL topologies are given in Sec.2.1. Helpful operation details for DHM, iSHM, the definition procedure, the class maps and the footprints of OV LV BPL topologies are reported in Secs 2.2-2.7, respectively.

\subsection{OV LV MTL Configuration and Indicative OV LV BPL Topologies}

As the BPL signal propagation is examined, the typical OV LV MTL configuration that is applied in this paper is shown in Fig. 1(a) of [1]. Note that the applied OV LV MTL configuration consists of four parallel non-insulated conductors (i.e., $n^{\mathrm{OVLV}}=4$ ), which are hung in vertical arrangement. More details concerning the MTL configuration dimensions, the conductor dimensions and the conductor structure are given in [18], [31] while the ground properties and the role of ground during the BPL signal propagation over OV power lines are detailed in [10], [18], [32]-[36].

As the BPL signal transmission is studied, the typical OV LV BPL topology that is used in this paper is shown in Fig. 1(b) of [1]. In fact, the examined typical OV LV BPL topology is the division result of OV LV BPL networks into simpler topologies of fixed end-to-end connection length (say, $1000 \mathrm{~m}$ in this paper) but different topological characteristics (say, number of branches, distances between branches and branch lengths) that depend on the underlying power grid. In accordance with [37], [38], [18], [39], five OV LV BPL topology classes (i.e., "LOS", rural, suburban, urban A and urban B) with their respective representative OV LV BPL topologies, which act as the real indicative OV LV BPL topologies of this paper, can be defined so that a classification of all OV LV BPL topologies (i.e., either real or virtual ones) can be made in terms of their capacity. In Table 1 of [1], the five real indicative OV LV BPL topologies of this paper are reported as well as their topological characteristics. 


\subsection{DHM}

With reference to BPMN diagrams of iSHM [1], [19], [21], it is evident that the numerical results of DHM act as the cornerstone of iSHM, class maps and footprints. DHM is a well validated model that has extensively been employed to examine the behavior of various transmission and distribution BPL networks [10], [11], [15]-[18], [32], [40]-[43]. In general terms, DHM consists of four interconnected submodules; say, two internal submodules (i.e., the bottom-up and top-down approach modules) and two external ones (i.e., the coupling scheme and the capacity computation modules). The numerical results of DHM that are of interest for iSHM and its tools of this paper are the transfer function and the capacity for given BPL topology, MTL configuration, coupling scheme, EMI policy and noise level. Mathematically, the output of the interconnection of the aforementioned internal submodules is the $n^{\text {ovLV }} \times n^{\text {ovLV }}$ line channel transfer function matrix

$$
\mathbf{H}^{\mathrm{OVLV}}\{\cdot\}=\mathbf{T}_{\mathrm{V}}^{\mathrm{OVLV}} \cdot \mathbf{H}^{\mathrm{OVLV}, \mathrm{m}}\{\cdot\} \cdot\left(\mathbf{T}_{V}^{\mathrm{OVLV}}\right)^{-1}
$$

where $\mathbf{H}^{\mathrm{OVLV}}, \mathrm{m}\{\cdot\}$ is the $n^{\mathrm{OVLV}} \times n^{\mathrm{OVLV}}$ modal channel transfer function matrix that mainly depends on the examined OV LV MTL configuration and OV LV BPL topology and $\mathrm{T}_{\mathrm{V}}^{\mathrm{OVLV}}$ is a $n^{\mathrm{OVLV}} \times n^{\mathrm{OVLV}}$ matrix that depends on the frequency, the physical properties of the TLs and the geometry of the OV LV MTL configuration. With reference to eq. (1), the output of the coupling scheme module is the coupling scheme channel transfer function that relates output and input BPL signal through

$$
H^{\mathrm{OVLV}, C}\{\cdot\}=\left[\mathbf{C}^{\text {out }}\right]^{\mathrm{OVLV}, C} \cdot \mathbf{H}^{\mathrm{OVLV}}\{\cdot\} \cdot\left[\mathbf{C}^{\mathrm{in}}\right]^{\mathrm{OVLV}, C}
$$

for given coupling scheme where []$^{c}$ denotes the applied coupling scheme among the supported coupling schemes of CS2 module [44], [45], $\mathbf{C}^{\text {in }}$ is the input coupling $n^{\mathrm{OVLV}} \times 1$ column vector that deals with the BPL signal injection interface and is defined by the applied coupling scheme and $\mathbf{C}^{\text {out }}$ is the ouput coupling $1 \times n^{\text {ovLV }}$ line vector that deals with the BPL signal extraction interface and is also defined by the applied coupling scheme. With reference to eq. (2), the output of the capacity computation module is the capacity $C$ that is given by [1], [37], [46], [47]

$$
\begin{gathered}
C=f_{s} \sum_{q=1}^{Q} \log _{2}\left\{1+\left[\frac{\left\langle p\left(f_{q}\right)\right\rangle_{\mathrm{L}}}{\left\langle N\left(f_{q}\right\rangle_{\mathrm{L}}\right.} \cdot\left|H^{\mathrm{OVLV}, \mathrm{C}}\left(f_{q}\right)\right|^{2}\right]\right\} \\
f_{q}=3 \mathrm{MHz}+(q-1) \cdot f_{s}, q=1, \ldots, Q \\
\mathbf{f}=\left[\begin{array}{lllll}
f_{1} & \cdots & f_{q} & \cdots & f_{Q}
\end{array}\right], q=1, \ldots, Q
\end{gathered}
$$

where $f_{q}$ is the flat-fading subchannel start frequency, $f_{s}$ is the flat-fading subchannel frequency spacing, $Q$ is the number of subchannels in the examined $3-30 \mathrm{MHz}$ frequency range, $\mathbf{f}$ is the $1 \times Q$ line vector that consists of the flat-fading subchannel start frequencies $f_{q}, p(\cdot)$ is the applied IPSD limits in $\mathrm{dBm} / \mathrm{Hz}, N(\cdot)$ is the applied AWGN PSD levels in $\mathrm{dBm} / \mathrm{Hz}$ and $\langle\cdot\rangle_{\mathrm{L}}$ is an operator that converts $\mathrm{dBm} / \mathrm{Hz}$ into a linear power ratio $(\mathrm{W} / \mathrm{Hz})$. More details concerning the applied coupling scheme, IPSD limits and AWGN PSD levels are given in [1], [2].

\section{3 iSHM}

Already been mentioned, SHM consists of iSHM and mSHM. With reference to the BPMN diagrams of iSHM and mSHM [1], [19], iSHM consists of six Phases (i.e., Phase A-F) while each Phase is clearly defined in terms of its procedure and the input / output files.

In accordance with [19] and with reference to Fig. 2(a) of [1], the operation of iSHM can be described through the concatenation of six Phases (i.e., Phases A-F). 
The input of iSHM, which coincides with the input parameters of its Phase A, is the topological characteristics of the examined real indicative OV LV BPL topologies, the applied coupling scheme, IPSD limits and AWGN PSD levels. The output of iSHM, which coincides with the output of Phase $F$, is the capacity range of each OV LV BPL topology main subclass for given CASD when each OV LV BPL topology main subclass consists of its representative real indicative OV LV BPL topology from Phase A and $P$ statistically equivalent virtual OV LV BPL topologies to the real indicative one in terms of CASD MLEs. Note that iSHM may support five CASDs with their corresponding MLEs (i.e., Gaussian, Lognormal, Wald, Weibull and Gumbel CASDs) while each CASD exhibits different performance depending on the input parameters of Phase A.

In accordance with [2], the performance of iSHM CASDs is assessed in terms of the best percentage change and average percentage change results that are performance capacity metrics. By evaluating the CASD approximation accuracy to the real capacity results, the best CASD is chosen for iSHM and are further applied during the definition procedure, class maps and footprints.

\subsection{The Definition Procedure and Class Maps}

As already been identified in [23], [24], [48], OV LV BPL topology classes are underrepresented since iSHM only exploits the five main OV LV BPL topology subclasses in order to define the respective classes for given CASD. The definition procedure, which has been introduced in [48], clearly solves this issue by statistically defining and inserting virtual indicative OV LV BPL topologies with their respective subclasses in the existing five OV LV BPL topology classes of iSHM by appropriately computing MLEs of iSHM CASDs. An appropriate flowchart that describes the definition procedure of iSHM for OV LV BPL networks is given in Fig. 3(a) of [1].

In accordance with [48], as the definition procedure of iSHM is concerned, it consists of eleven steps (i.e., FL1.01-FL1.11) that can be further categorized into three groups (i.e., Group 1.A-1.C). The input of the iSHM definition procedure is the five main OV LV BPL topology subclasses with their respective representative real indicative OV LV BPL topologies for given CASD. The output of the iSHM definition procedure is the class map that is a 2D contour plot where (i) each CASD parameter pair is corresponded to its OV LV BPL topology subclass average capacity; (ii) real and virtual OV LV BPL topology subclasses are described by corresponding CASD parameter pairs; (iii) the borders between the OV LV BPL topology classes are illustrated; and (iv) by taking into the capacity arrangement of OV LV BPL topology subclasses and the borders between the OV LV BPL topology classes, OV LV BPL topology class areas can be demonstrated.

In Secs 2.3 and 2.4, DHM, iSHM, definition procedure and class maps have been presented that form all together an interconnected system but with specified settings. The set of the default operation settings that are used in this paper are simply reported in Sec3.4 of [1] as a whole.

\section{5 iSHM Footprints of Real OV LV BPL Topologies on Class Maps}

The theoretical definition of footprints has been presented in [1] while the first numerical results concerning the application of footprints during critical events of the operation of OV LV power grids have been demonstrated in [2], say, the iSHM footprints 
of the real OV LV BPL topologies, of the OV LV BPL topologies with a sole branch line fault and of the OV LV BPL topologies with a single hook for energy theft.

As the iSHM footprints of the real OV LV BPL topologies are of interest in this paper, their computation is based on TIM that allows the accurate identification of an OV LV BPL topology with respect to its topological characteristics (i.e., number of branches, length of branches, length of main distribution lines and branch terminations) by applying piecewise monotonic data approximations even if significant measurement differences may occur [49]-[51]. In fact, the computation of footprints is feasible by exploiting: (i) the OV LV BPL topology database of TIM that consists of OV LV BPL topologies with their respective theoretical and measured coupling scheme channel transfer functions when appropriate TIM BPL topology database specifications (e.g., the maximum number of branches, the length spacing for both branch distance and branch length and the maximum branch length) are assumed; and (ii) the computation of CASD MLEs of each OV LV BPL topology of the TIM BPL topology database for given best CASD for the preparation of the iSHM footprint [1]. As been demonstrated in [2], iSHM footprints of the real OV LV BPL topologies without measurement differences can be shown as areas of superimposed white spots upon the iSHM class maps for given power grid type, CASD, coupling scheme, IPSD limits and noise limits. By studying iSHM footprints of the real OV LV BPL topologies of [2], it is clear that the footprint extent of the real OV LV BPL topologies remains a small area of the respective class maps thus highlighting the significant and successful role of the respective definition procedures towards the enrichment of OV LV BPL topology classes with virtual OV LV BPL topologies.

Although iSHM footprints of the real OV LV BPL topologies graphically reveal the internal zones of the respective class maps where real OV LV BPL topologies can be located, the measurement differences during the computation of the coupling scheme transfer functions have been ignored in [2]. In this paper, the option of the TIM OV LV BPL topology database that considers measurement differences during the computation of coupling scheme transfer functions is activated thus allowing the impact assessment of measurement differences when various distributions are applied. In the following Section, the mathematics of the measurement difference distributions are reported as well as their interaction with DHM, iSHM, definition procedures, class maps and footprints. Note that although default operation settings have been assumed in Sec 2.4 of [2] for the interaction of iSHM footprints with the remaining set (i.e., DHM, iSHM, definition procedures and class maps), there are no need for these assumptions here since the impact of measurement differences is going be studied with reference to the five real indicative OV LV BPL topologies of Table 1 of [1].

\section{The Involvement of Measurement Differences from DHM to iSHM Footprints}

In this Section, the way the measurement differences are taken into account in DHM are presented. In addition, the approach and the settings for the study of iSHM footprints when measurement differences are applied are reported.

\subsection{Measurement Differences in DHM}

From DHM to iSHM footprints, the theoretical computation of the coupling scheme channel transfer function, which is mathematically described in eq. (2), has been 
applied so far since no measurement differences have been assumed. However, a set of practical reasons and "real-life" conditions, which can be grouped into six categories [25], [27], [52], are taken into account in this paper so that the role and the effect of measurement differences can be further investigated. In accordance with [25], [27], [52] and eq. (2), the measured coupling scheme transfer function $\overline{H_{d 1, d 2, l}^{\mathrm{OVLV}, C, D}}\{\cdot\}$ after the consideration of measurement differences is determined by

$$
\overline{H_{d 1, d 2, l}^{\mathrm{OVLV}, D}}\left(f_{q}\right)=H^{\mathrm{OVLV}, C}\left(f_{q}\right)+e_{d 1, d 2, i}^{D}\left(f_{q}\right), q=1, \ldots, Q, i=1, \ldots, I
$$

where $[\cdot]^{D}$ denotes the applied measurement difference distribution -either CUD or ND-, $d 1$ is the first parameter of the applied measurement difference distribution (the minimum value $-a_{\text {CUD }}$ or the mean $\mu_{\mathrm{ND}}$ of CUD or ND, respectively), $d 2$ is the second parameter of the applied measurement difference distribution (the maximum value $a_{\text {CUD }}$ or the standard deviation $\sigma_{\mathrm{ND}}$ of CUD or $\mathrm{ND}$, respectively), $e_{d 1, d 2, i}^{D}\left(f_{q}\right)$ is the measurement difference at frequency $f_{q}$ for given measurement difference distribution, $I$ is the number of different $1 \times Q$ line vectors of measurement differences per applied measurement difference distribution, first and second parameter and $i$ indicates the $i^{\text {th }}$ among $I$ line vectors of measurement differences. In this paper, 100 line vectors of measurement differences are going to be assumed per applied measurement difference distribution, first and second parameter $(I=100)$.

\subsection{Measurement Differences and Settings for the SHM Footprints}

In order to highlight the impact of measurement differences on iSHM footprints for the five real indicative OV LV BPL topologies of Table 1 of [1], the iSHM class map that is shown in Fig. 1 of [2] is adopted as the graphical basis. With reference to the five real indicative OV LV BPL topologies of Table 1 of [1] and the BPMN diagrams of iSHM [1], [19], it is evident that the applied theoretical coupling scheme transfer functions are going to be replaced by the measured ones, which are described in eq. (6), thus directly affecting the output of DHM module of Phase A of the iSHM BPMN diagrams -see Fig. 2(a) of [1], respectively-.

To illustrate the impact of each line vector of measurement differences on the class maps of each real OV LV BPL topology of Table 1 of [1], the corresponding measured coupling scheme transfer function is accompanied by: (i) the respective MLEs of the Weibull CASD as described in iSHM BPMN diagram Phase C of Fig. 2(a) of [1] (i.e., MLE computation module). Here, it should be noted that the Weibull CASD has been verified to be the best CASD for OV LV BPL topologies in [2]; and (ii) the capacity; when the default operation settings of Sec.3.4 of [1] are assumed.

As the iSHM footprint of each real OV LV BPL topology of Table 1 of [1] is considered for given measurement difference distribution, first and second parameter, the same procedure that is presented in [1] for the iSHM footprint of real OV LV BPL topologies is also followed here but with the following two differences: (i) Only the five real OV LV BPL topologies of Table 1 of [1] are used and not the entire TIM OV LV BPL topology database; and (ii) for each of the five real OV LV BPL topologies of Table 1 of [1], 100 white spots are expected to appear on Weibull CASD class maps as footprint that correspond to the respective combination of Weibull CASD MLEs and capacity of the 100 line vectors of measurement differences that are superimposed to the coupling scheme transfer function of each of the five real OV LV BPL topologies of Table 1 of [1]. 


\section{Numerical Results and Discussion}

In this Section, numerical results assessing the impact of measurement differences on iSHM footprints of OV LV BPL topologies are demonstrated. First, the effect of the different measurement distributions (i.e., CUD or ND) is highlighted through the iSHM footprints for the five real OV LV BPL topologies of Table 1 of [1]. Second, the sensitivity of the aforementioned real OV LV BPL topologies to the measurement differences is revealed through their iSHM footprints. Third, for given real OV LV BPL topology, the effect of the intensity of measurement differences, say, the change of the first and second parameter values of the CUD and ND measurement distributions, on iSHM footprints is revealed.

\section{1 iSHM Class Maps of OV LV BPL Topologies}

In accordance with the iSHM definition [1], [19], [20], the CASD MLEs of iSHM are computed at the Phase C of Fig. 2(a) of [1]. As the default operation settings have been assumed in [1], MLEs of the Gaussian, Lognormal, Wald, Weibull and Gumbel CASDs of iSHM have been reported in Table 1 of [2] for the main subclasses of the real indicative OV LV BPL topologies of Table 1 of [1]. Their capacity estimation performance of CASDs of iSHM has been benchmarked via the percentage change and the average absolute percentage change of Table 2 of [2]. In accordance with Table 2 of [2], Weibull CASD performs the best performance among the available iSHM CASDs with respect to the percentage change and average absolute percentage change in all the examined OV LV BPL topology main subclasses. Therefore, only Weibull CASD is going to be applied during the following subsections. In accordance with [2], [23], [24], the iSHM class map of OV LV BPL topologies is plotted in Fig. 1 with respect to

$\hat{a}_{\mathrm{MLE}}^{\mathrm{Weibull}}, \hat{\beta}_{\mathrm{MLE}}^{\mathrm{Weibull}}$ and the average capacity of each OV LV BPL topology subclass.

\section{2 iSHM Footprints of Measurement Differences - The Effect of the Different Distributions of Measurement Differences on OV LV BPL Topologies}

The impact of measurement differences on iSHM class maps of OV LV BPL topologies is examined via footprints. Actually, the effect of the different distributions of measurement differences (i.e., CUD or ND) and the behavior of each of the real indicative OV LV BPL topologies when measurement differences are applied are going to be studied in this subsection. With reference to the iSHM class map of Fig. 1, the iSHM footprint of the real indicative OV LV BPL urban case A is illustrated as superimposed white spots on class maps in Fig. 2(a). Note that the CUD measurement difference of maximum value $a_{\mathrm{CUD}}$ of $3 \mathrm{~dB}$ and 100 line vectors of random measurement differences of the aforementioned CUD are assumed during the preparation of Fig. 2(a). In Figs. 2(b)-(e), similar footprints with Fig. 2(a) are illustrated but for the case of urban case B, suburban case, rural case and "LOS" case, respectively, when the same 100 line vectors of measurement differences are applied. In Figs. 3(a)-(e), similar footprints with Figs. 2(a)-(e) are given but for the case of ND measurement differences of mean $\mu_{\mathrm{ND}}$ and standard deviation $\sigma_{\mathrm{ND}}$ that are equal to $0 \mathrm{~dB}$ and $3 / 2 \mathrm{~dB}$, respectively. Note that the same 100 line vectors of ND measurement differences are applied among Figs. 3(a)-(e). 


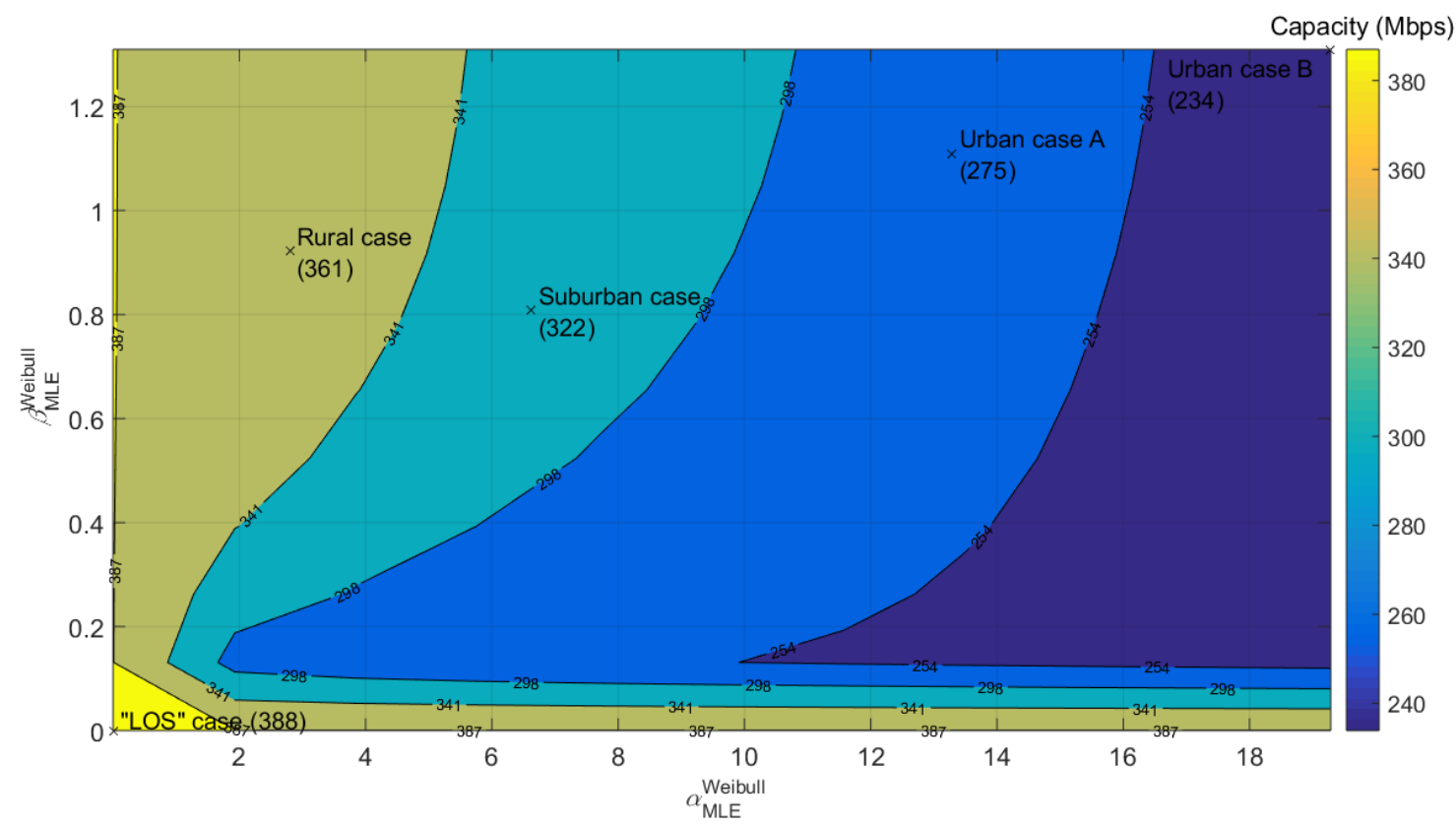

Fig. 1. iSHM class map of OV LV BPL topologies when $3-30 \mathrm{MHz}$ frequency band, $\mathrm{WtG}^{1}$ coupling scheme and FCC Part 15 are assumed [2]. 


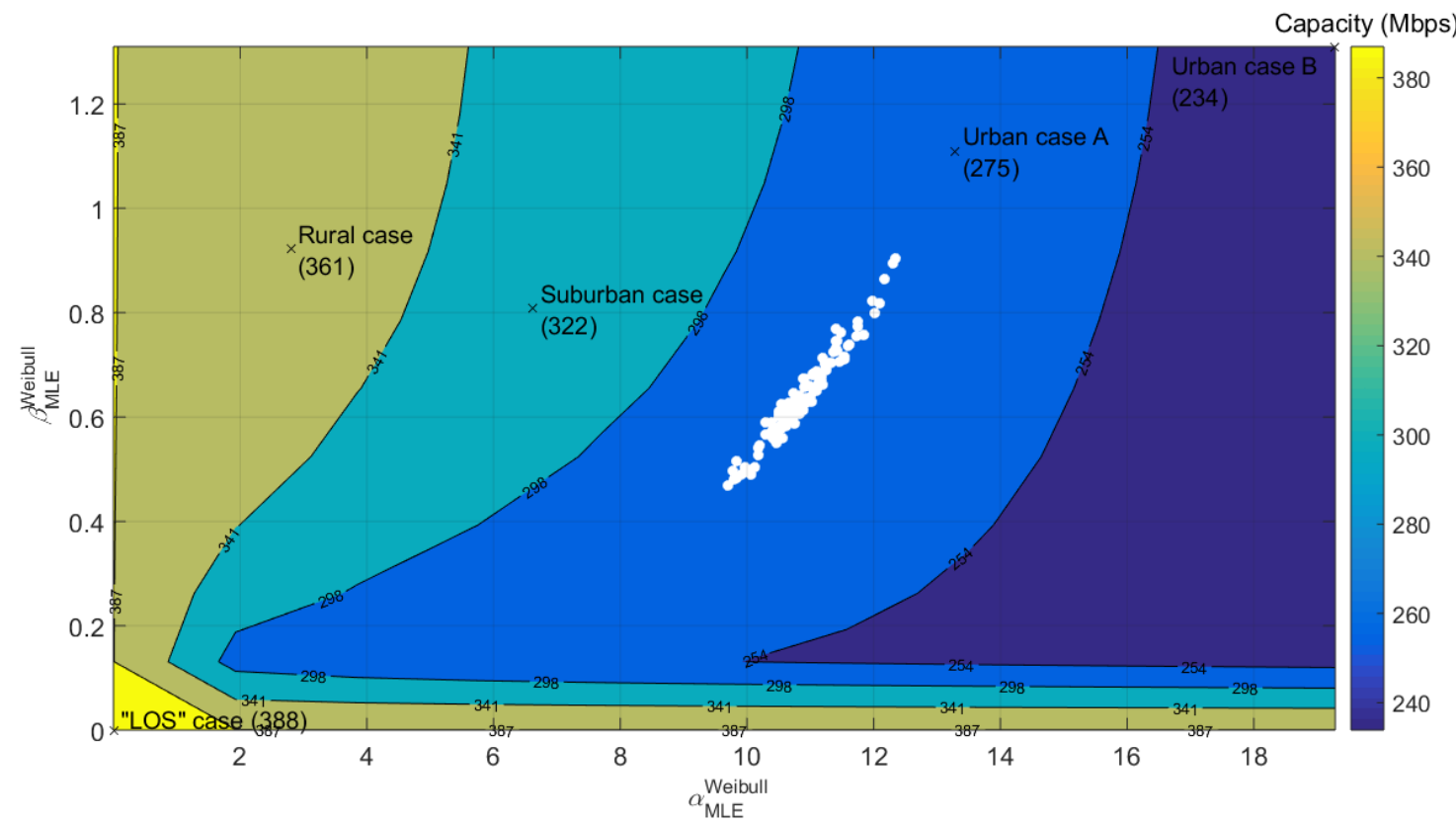

(a)

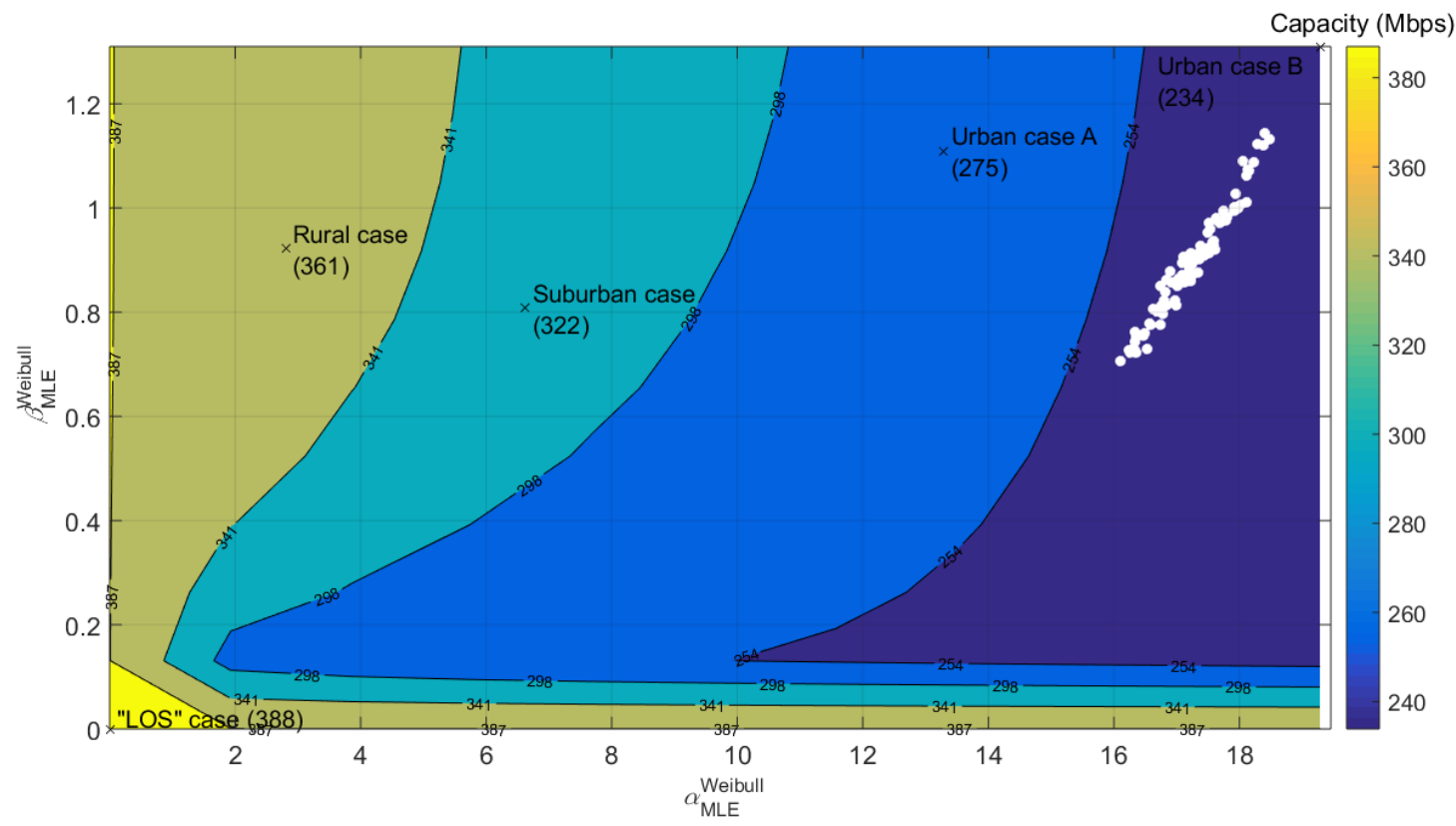

(b) 


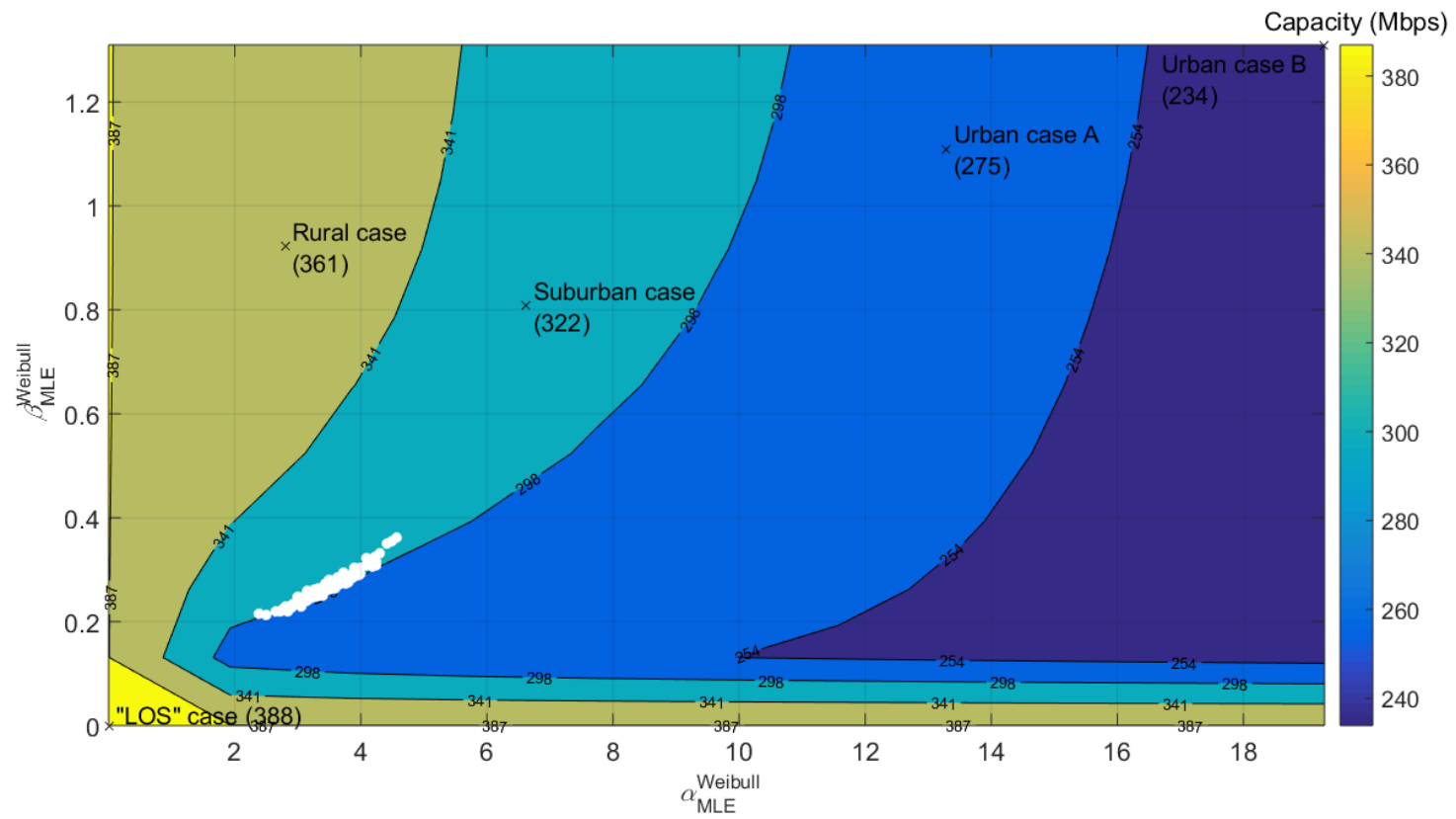

(c)

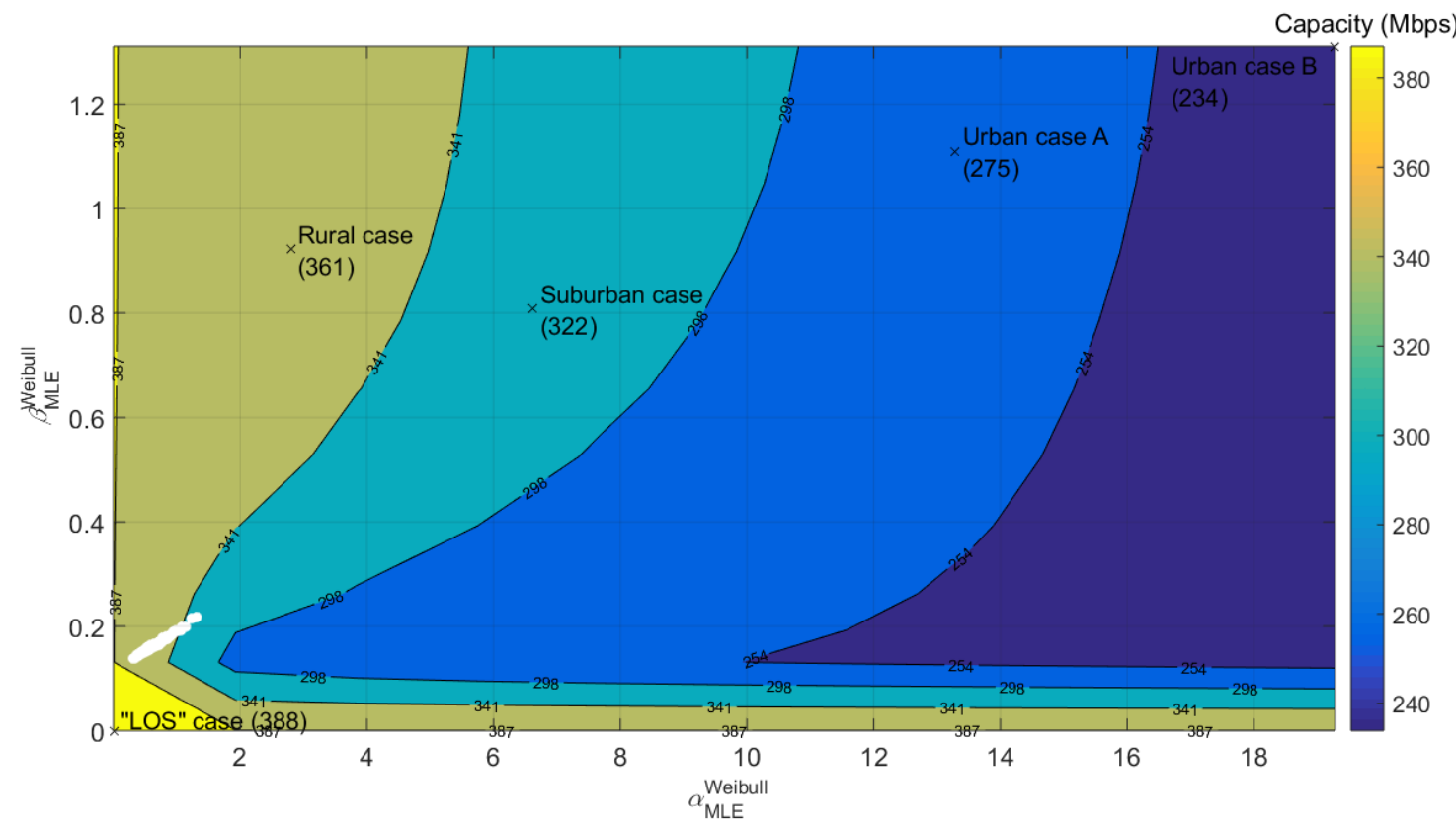

(d) 


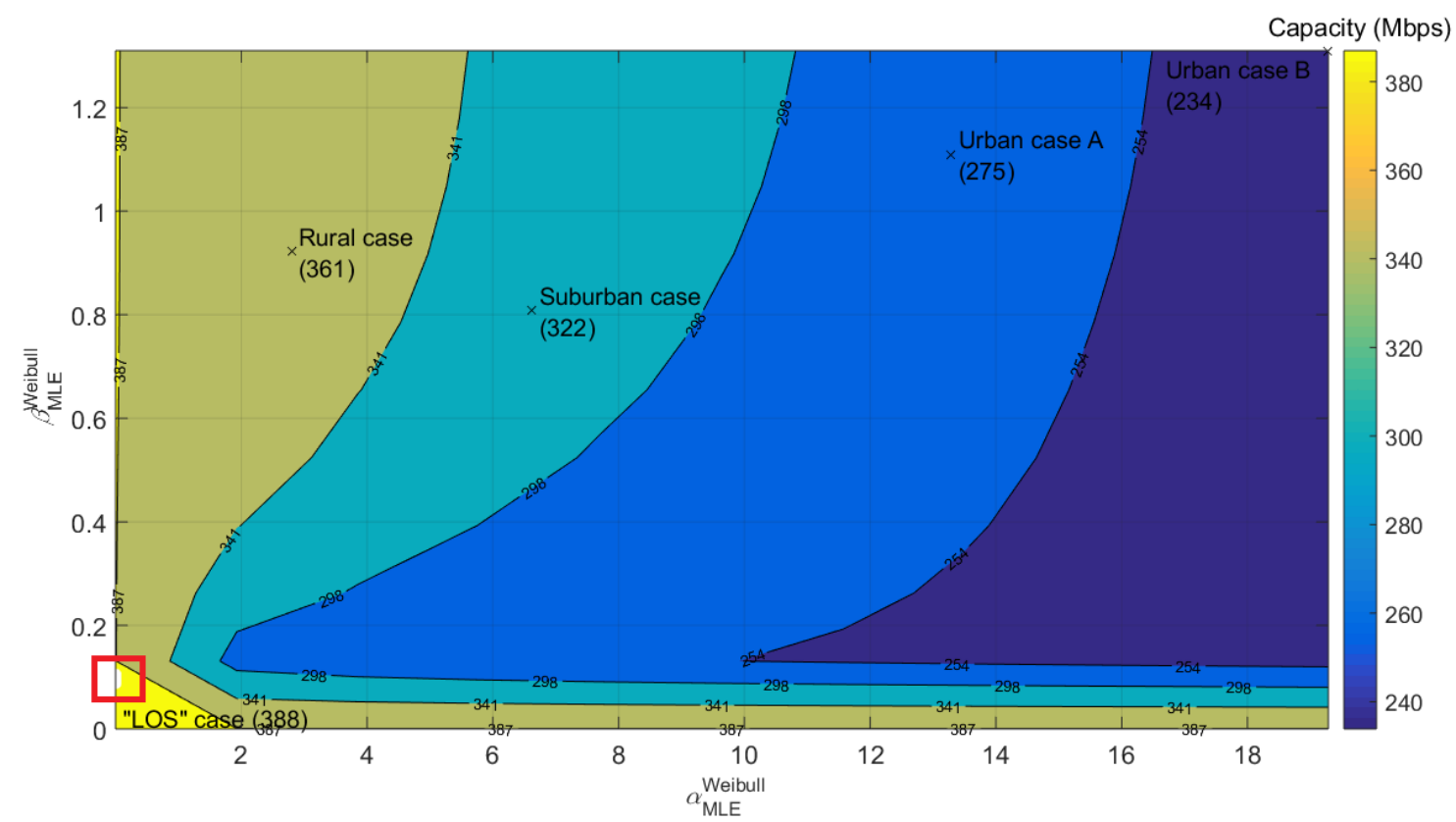

(e)

Fig. 2. iSHM footprints of the real indicative OV LV BPL topologies when $3-30 \mathrm{MHz}$ frequency band, $\mathrm{WtG}^{1}$ coupling scheme, FCC Part 15 and CUD measurement differences of maximum value $a_{\mathrm{CUD}}=3 \mathrm{~dB}$ are assumed. (a) Urban case A. (b) Urban case B. (c) Suburban case. (d) Rural case. (e) "LOS" case. 


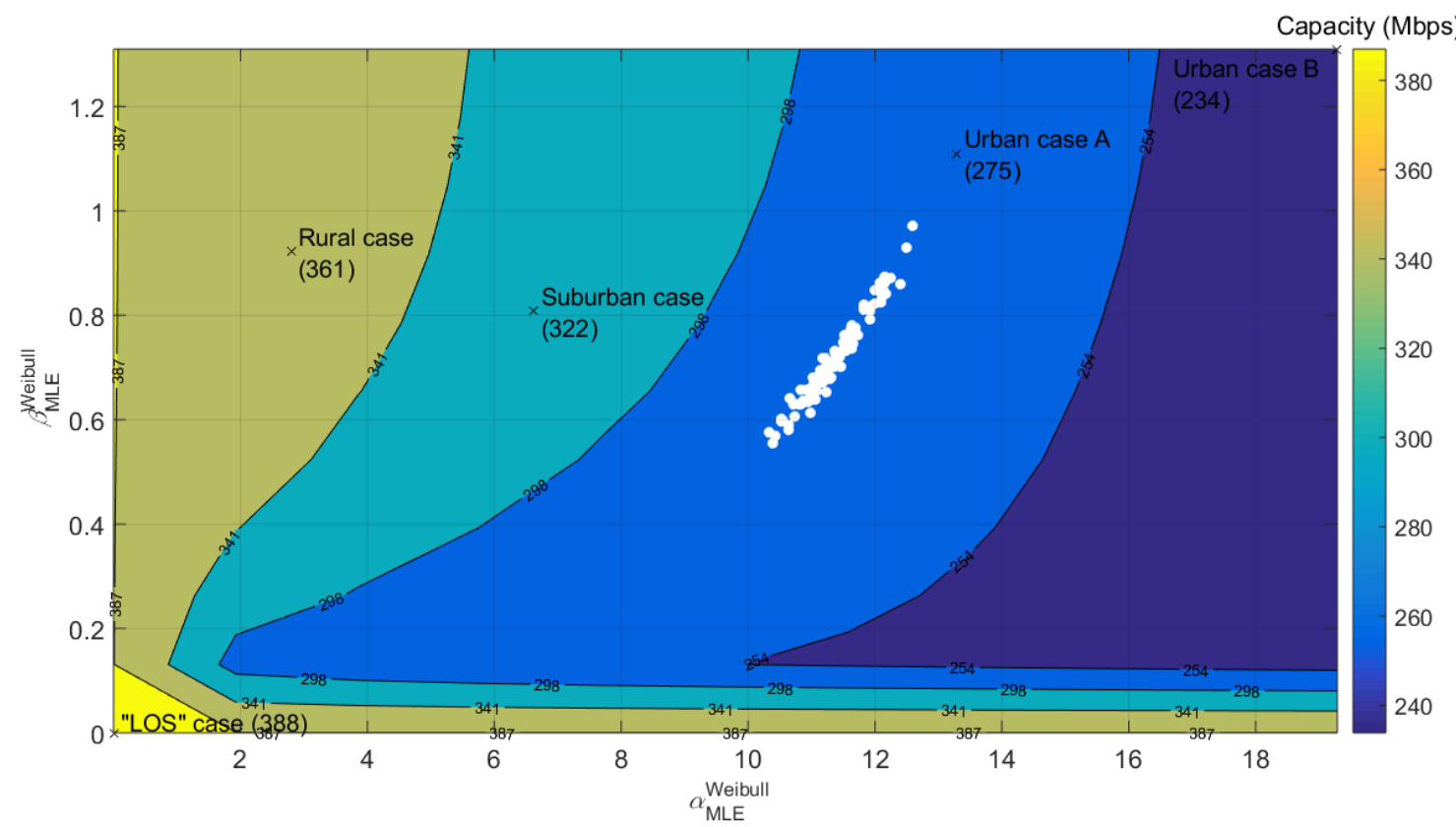

(a)

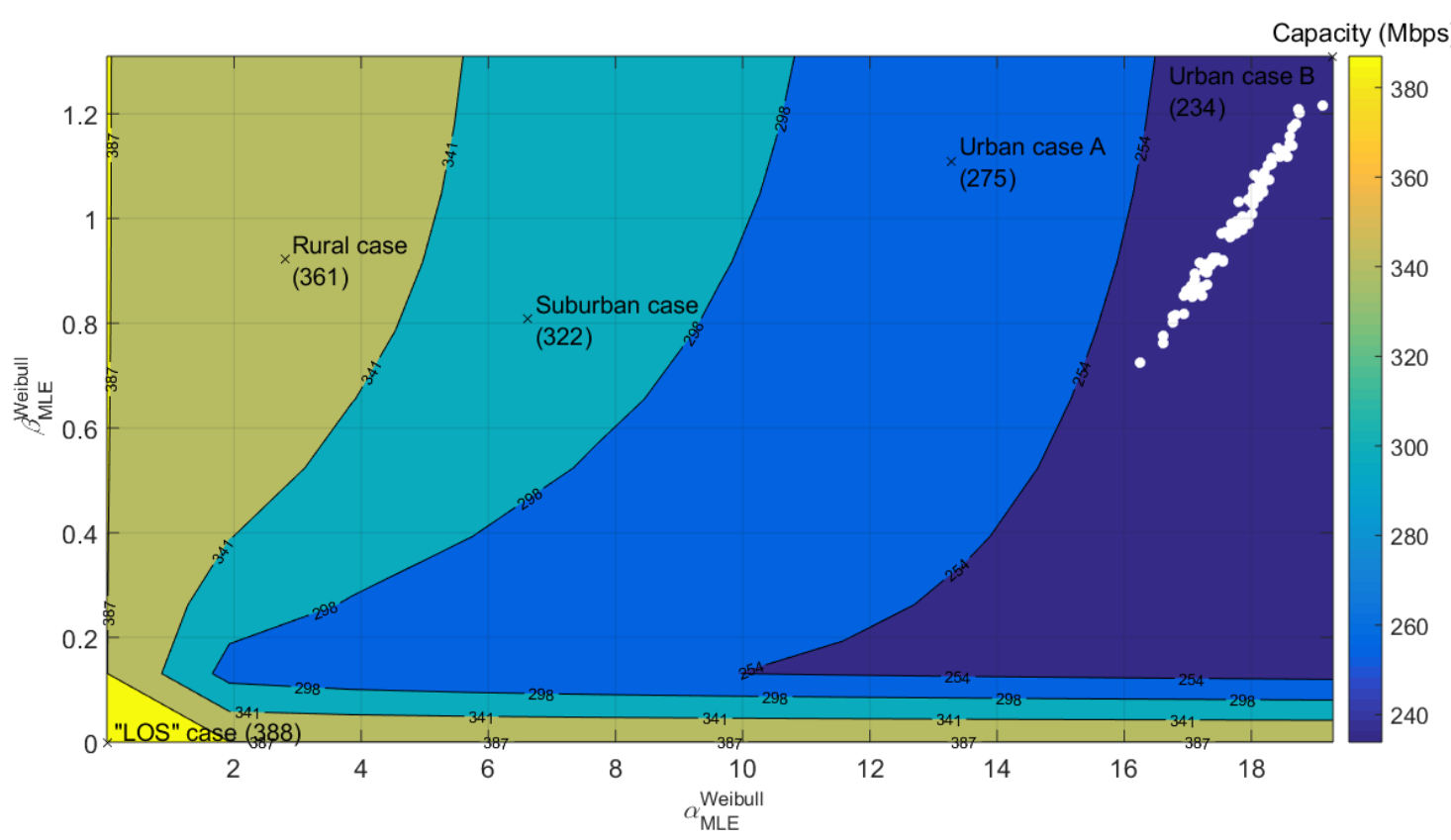

(b) 


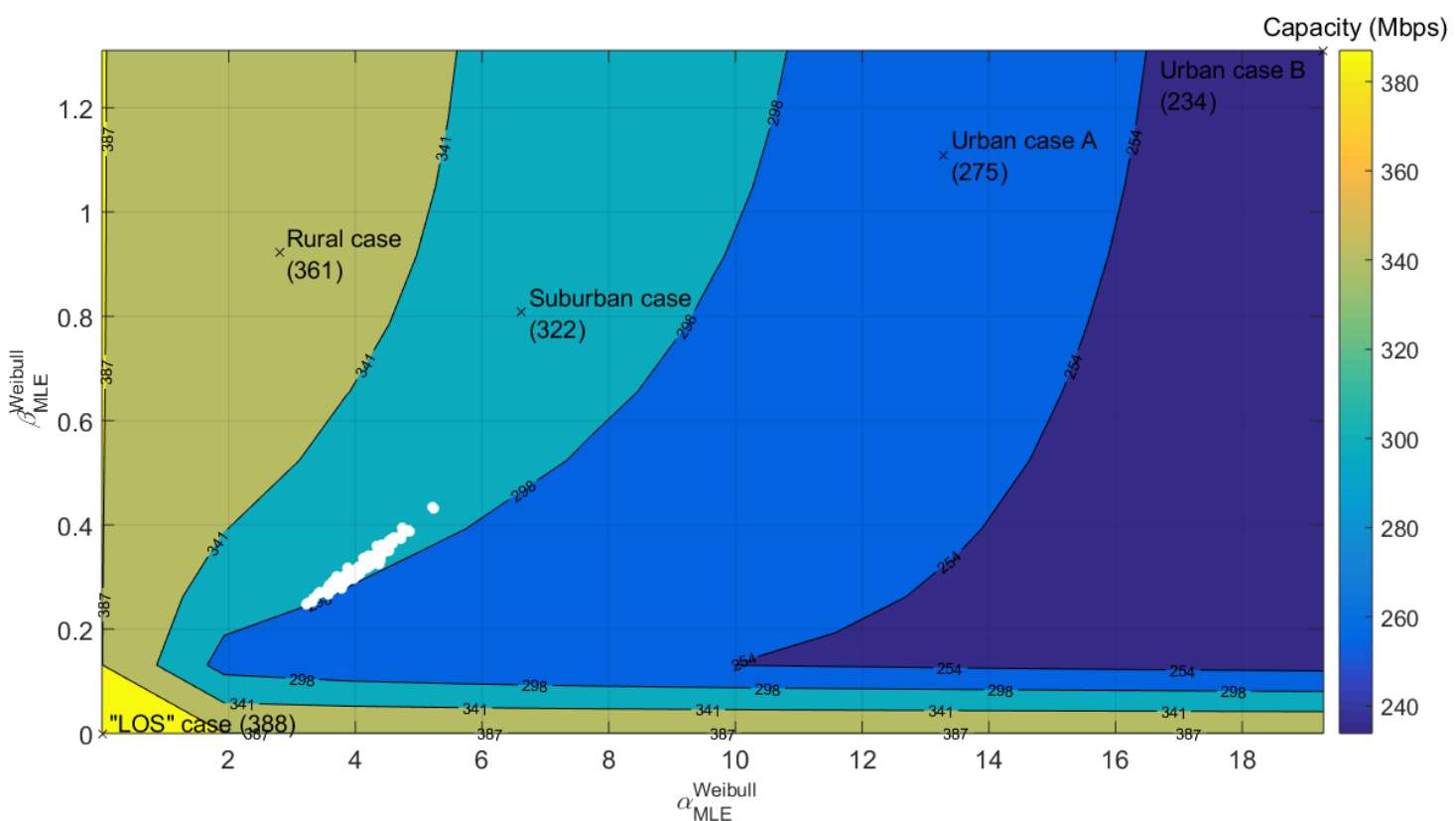

(c)

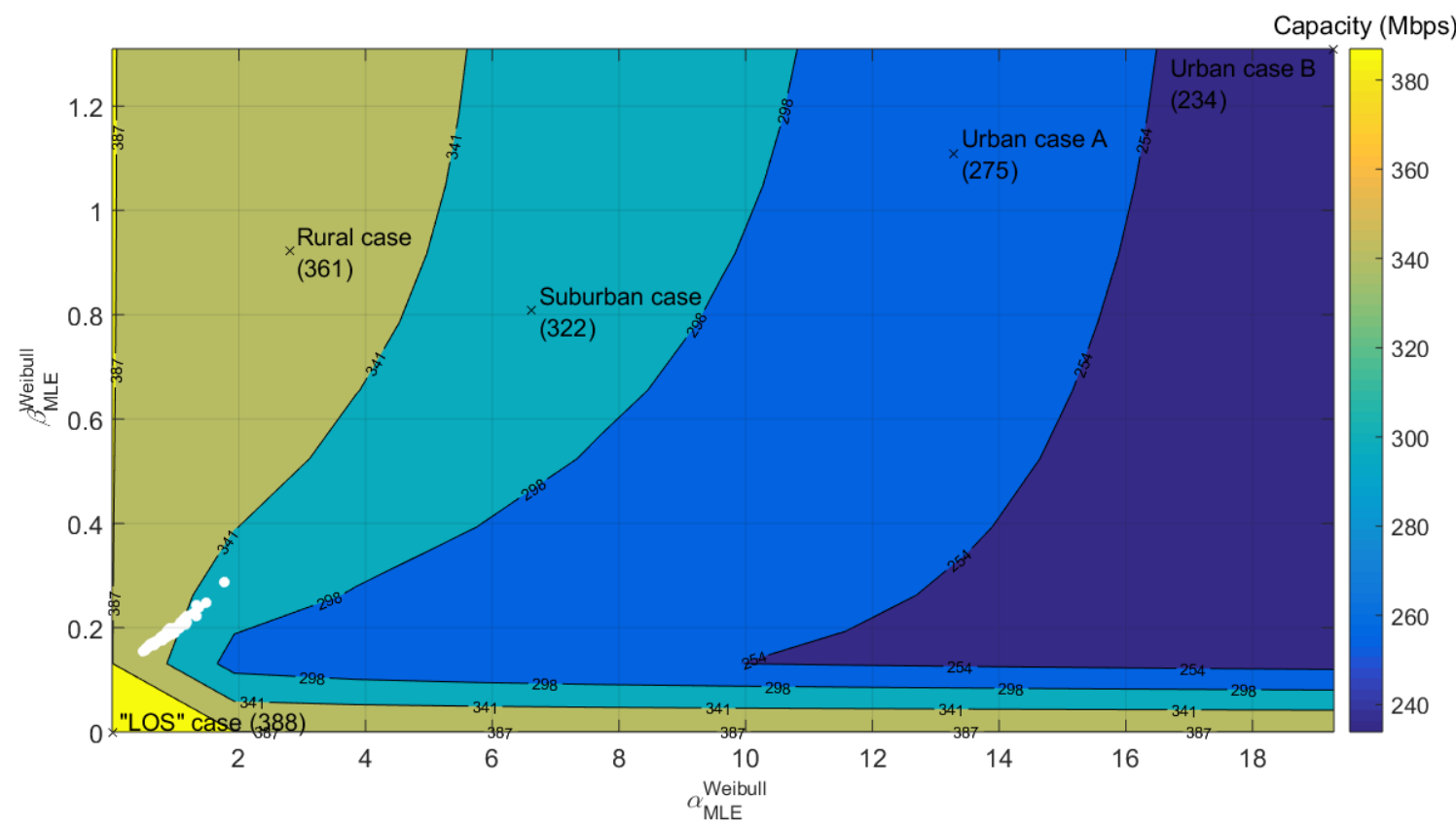

(d) 


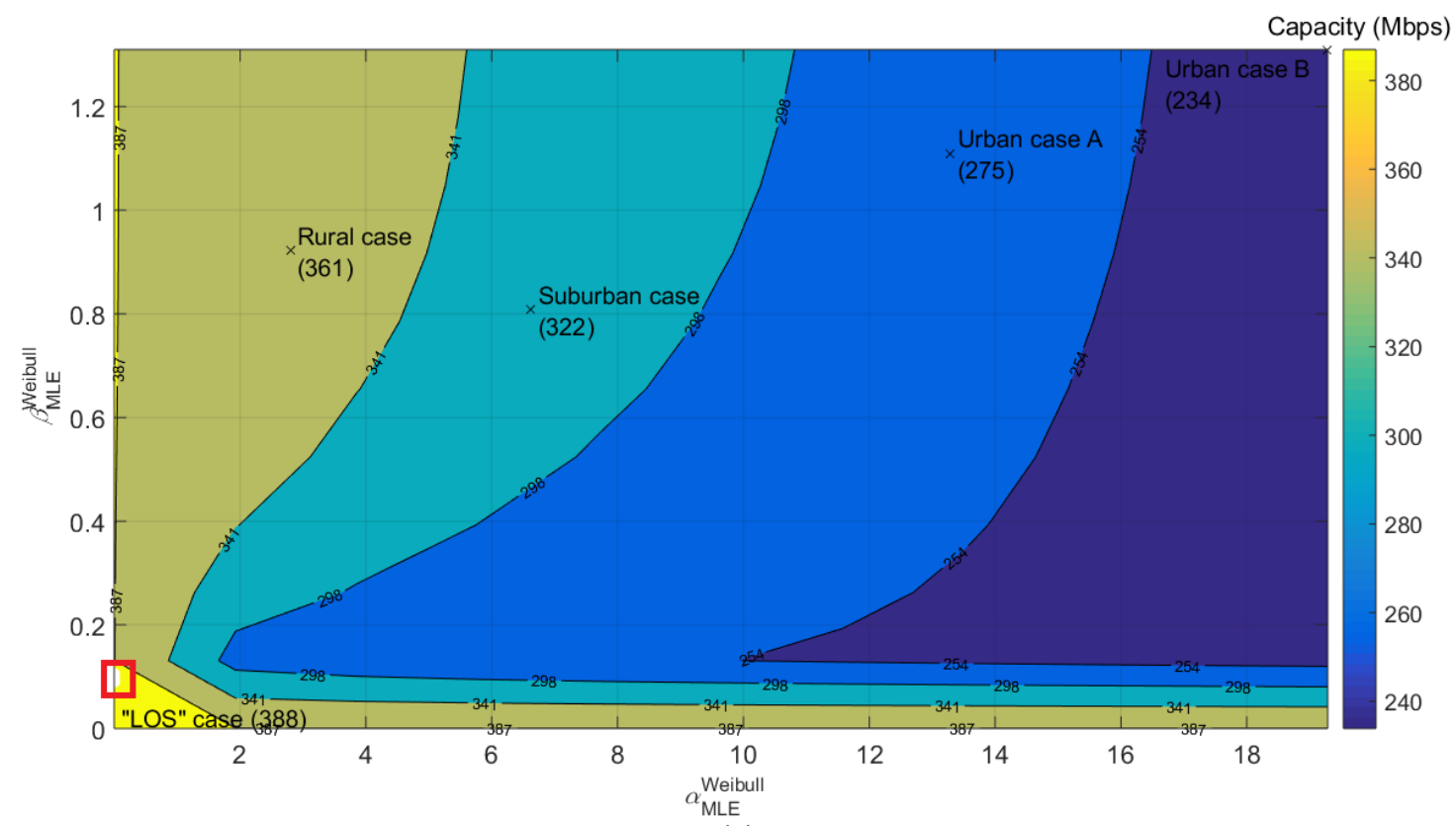

(e)

Fig. 3. Same plots but for ND measurement difference of mean $\mu_{\mathrm{ND}}=0 \mathrm{~dB}$ and standard deviation $\sigma_{\mathrm{ND}}=3 / 2 \mathrm{~dB}$.

By observing Figs. 2(a)-(e) and 3(a)-(e), several interesting observations can be made regarding the presence of measurement differences, namely:

- By comparing the aforementioned figures with Figs. 3(a) and 3(b) of [2], it is evident that the iSHM footprint behavior when measurement differences are applied present similarities to the behavior of OV LV BPL topologies with short branches for given OV LV BPL topology class. With reference to the theoretical values of $\hat{a}_{\mathrm{MLE}}^{\mathrm{Weibull}}$ and $\hat{\beta}_{\mathrm{MLE}}^{\mathrm{Weibull}}$ of the real indicative OV LV BPL topologies that act as the representative ones of classes, measurement differences tend to reduce the theoretical values of $\hat{a}_{\mathrm{MLE}}^{\mathrm{Weibull}}$ and $\hat{\beta}_{\mathrm{MLE}}^{\mathrm{Weibull}}$ of the representative OV LV BPL topologies thus forming the iSHM footprint as a diagonal white area of 100 white spots that is located at the lower left area of the examined class with approximate direction to the axis origin.

- As the location and the extent of the iSHM footprint are concerned for given OV LV BPL topology class, the footprint remains almost the same when the standard deviation $\sigma_{\mathrm{ND}}$ of ND measurement differences is approximately equal to the half of the maximum value $a_{\text {CUD }}$ of CUD measurement differences while the mean value $\mu_{\mathrm{ND}}$ of ND measurement differences is equal to zero. This fact can be explained by the distribution of PDFs of the previous CUD and ND measurement differences. Note that the mean $\mu_{\mathrm{ND}}$ of ND measurement differences is assumed to be equal to 0 , hereafter.

- As the maximum value $a_{\text {CUD }}$ of CUD measurement differences and the standard deviation $\sigma_{\mathrm{ND}}$ of ND measurement differences remain low -say, Figs. 2(a)-(e) and 3(a)-(e)-, the footprint remains within the class area boundaries for given real indicative OV LV BPL topology. 
- The direction of the footprint as well as its location can be explained by the management of the measurement differences with respect to the measured coupling scheme channel attenuation difference between each measured OV LV BPL topology and its respective theoretical "LOS" case [19]. More analytically, as the measured coupling scheme channel attenuation difference between each examined OV LV BPL topology and its respective theoretical "LOS" case is concerned, this should be remain greater or equal to zero (zero restriction of the measured coupling scheme channel attenuation difference). The zero restriction of the measured coupling scheme channel attenuation difference is a crucial condition for the fine iSHM procedure so that the MLE computation module of Phase $\mathrm{C}$ of iSHM BPMN diagram can operate and produce the Weibull MLEs of the examined OV LV BPL topology [19]. In accordance with [20], the coupling scheme channel attenuation differences are assumed to be equal to an arbitrarily low value, say $1 \times 10^{-11}$, instead of zero so that MLEs of Weibull CASD, which comprise natural logarithms and denominators, can be calculated.

- Depending on the intensity of measurement differences and the examined real indicative OV LV BPL topology, the zero restriction of the measured coupling scheme channel attenuation difference has as a result the greater concentration of channel attenuation difference values at zero that further affects the Weibull CASD approximation and its corresponding MLEs. In fact, as more channel attenuation difference values are located at zero, the approximated Weibull PDF tends to increase its maximum PDF value and to shift he location of the maximum PDF left that further entail simultaneously lower values of $\hat{a}_{\mathrm{MLE}}^{\mathrm{Weibull}}$ and $\hat{\beta}_{\mathrm{MLE}}^{\mathrm{Weibull}}$ with respect to the theoretical values of $\hat{a}_{\mathrm{MLE}}^{\mathrm{Weibull}}$ and $\hat{\beta}_{\mathrm{MLE}}^{\mathrm{Weibull}}$ for given real indicative OV LV BPL topology.

- The aforementioned zero restriction of the measured coupling scheme channel attenuation difference explains the dependence of the extent of the footprint on the multipath aggravation of the examined real indicative OV LV BPL topologies. Since coupling scheme transfer functions of less aggravated OV LV BPL topologies (i.e., suburban and rural case) are closer to the "LOS" case, the zero restriction of the measured coupling scheme channel attenuation difference may be activated more frequently than in the cases of aggravated OV LV BPL topologies (i.e., urban case A and B) when measurement differences are applied. Struggling to approximate the greater number of zero channel attenuation differences, the deeper notches due to the remaining measurement differences for given line vector have little effect to the approximated Weibull PDFs and, hence, small differences are expected to the Weibull CASD MLEs for the assumed line vectors of measurement differences thus entailing reduced sizes of the footprints of the less aggravated OV LV BPL topologies.

- As the OV LV BPL "LOS" case is examined as well as its iSHM footprint behavior when measurement differences are applied, the iSHM footprint of "LOS" case is located very close to the axes origin while its extent remains the smallest among the examined ones of real indicative OV LV BPL topologies. As measurement differences are superimposed to the coupling scheme channel attenuation of OV LV BPL "LOS" case, this has as effect almost the half of the measured coupling scheme channel attenuation difference values between the 
measured OV LV BPL "LOS" case and the theoretical "LOS" case be equal to zero due to the zero restriction of the measured coupling scheme channel attenuation difference and the other half of the measured coupling scheme channel attenuation difference values be affected by the measurement differences. The latter values are responsible for the little shift of the iSHM footprint of "LOS" case with reference to the axis origin.

Until now, the impact of measurement differences on class maps of the real indicative OV LV BPL topologies has been investigated in terms of the relative location and the extent of the corresponding iSHM footprints. The most important characteristic of measurement differences that is their intensity is studied in the following subsection.

\section{3 iSHM Footprints of Measurement Differences - The Effect of the Different Intensities of Measurement Differences on OV LV BPL Topologies}

Higher intensities of measurement differences imply higher maximum values $a_{\text {CUD }}$ and standard deviations $\sigma_{\mathrm{ND}}$ when CUD and ND measurement differences are applied, respectively. With reference to the iSHM class map of Fig. 1, the iSHM footprint of the real indicative OV LV BPL topology of urban case A is illustrated as superimposed spots on class maps in Fig. 4(a) when the maximum value $a_{\text {CUD }}$ of CUD measurement differences ranges from $0 \mathrm{~B}$ to $15 \mathrm{~dB}$. Conversely to Fig. 2(a), only one random line vector of measurement differences is applied for each maximum value $a_{\mathrm{CUD}}$ while the color of superimposed spots becomes redder as the maximum value $a_{\text {CUD }}$ increases. In Figs. 4(b)-(e), similar footprints with Fig. 4(a) are illustrated but for the case of urban case B, suburban case, rural case and "LOS" case, respectively, when the same line vectors of measurement differences per maximum value $a_{\text {CUD }}$ are applied. In Figs. 5(a)-(e), similar footprints with Figs. 4(a)-(e) are given but for the case of ND measurement differences where the mean $\mu_{\mathrm{ND}}$ is equal to $0 \mathrm{~dB}$ and the standard deviation $\sigma_{\mathrm{ND}}$ ranges from $0 \mathrm{~dB}$ to $15 / 2 \mathrm{~dB}$, respectively. Note that the same line vectors of ND measurement differences per standard deviation $\sigma_{\mathrm{ND}}$ are applied in Figs. 5(a)-(e). 


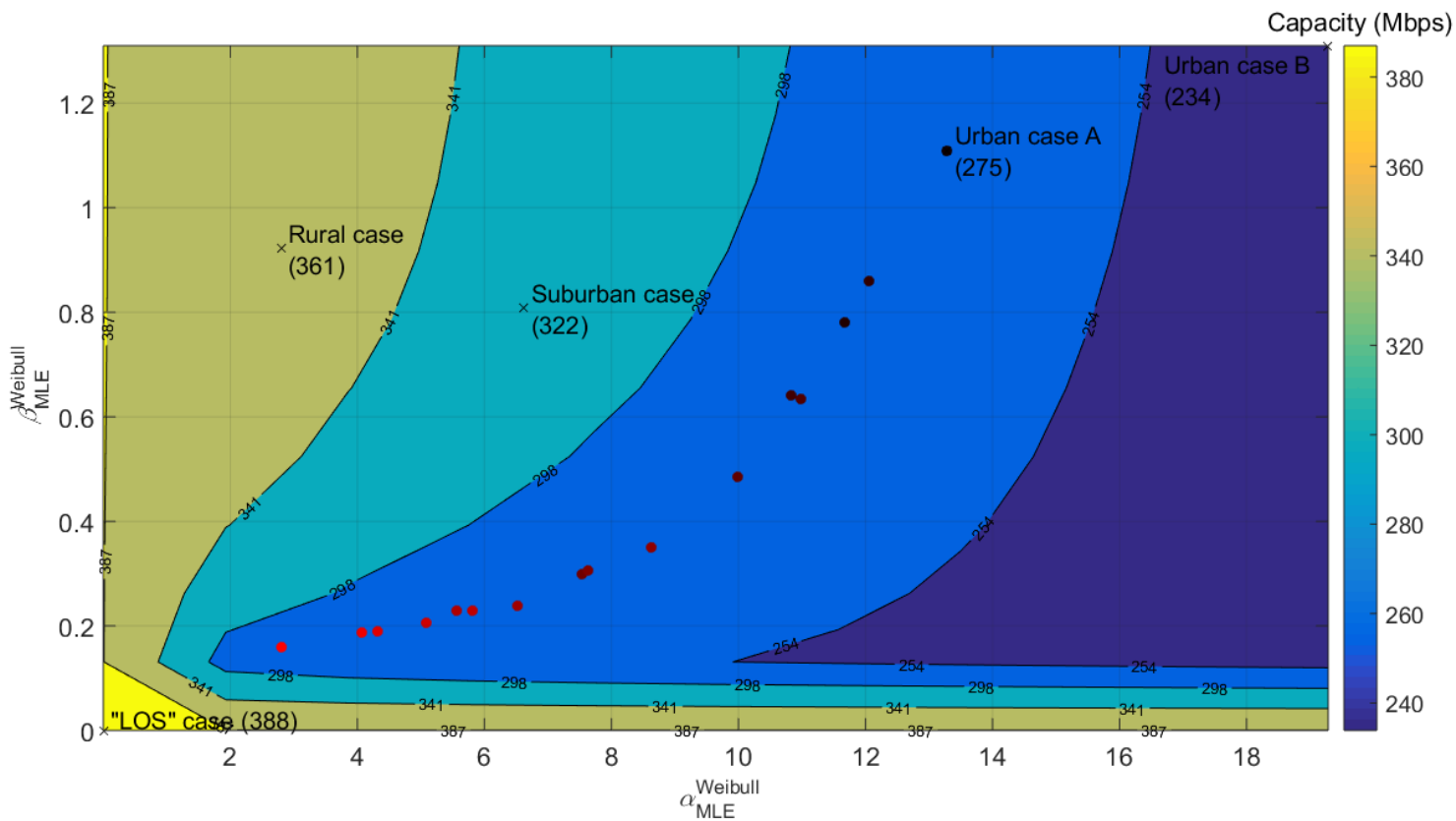

(a)

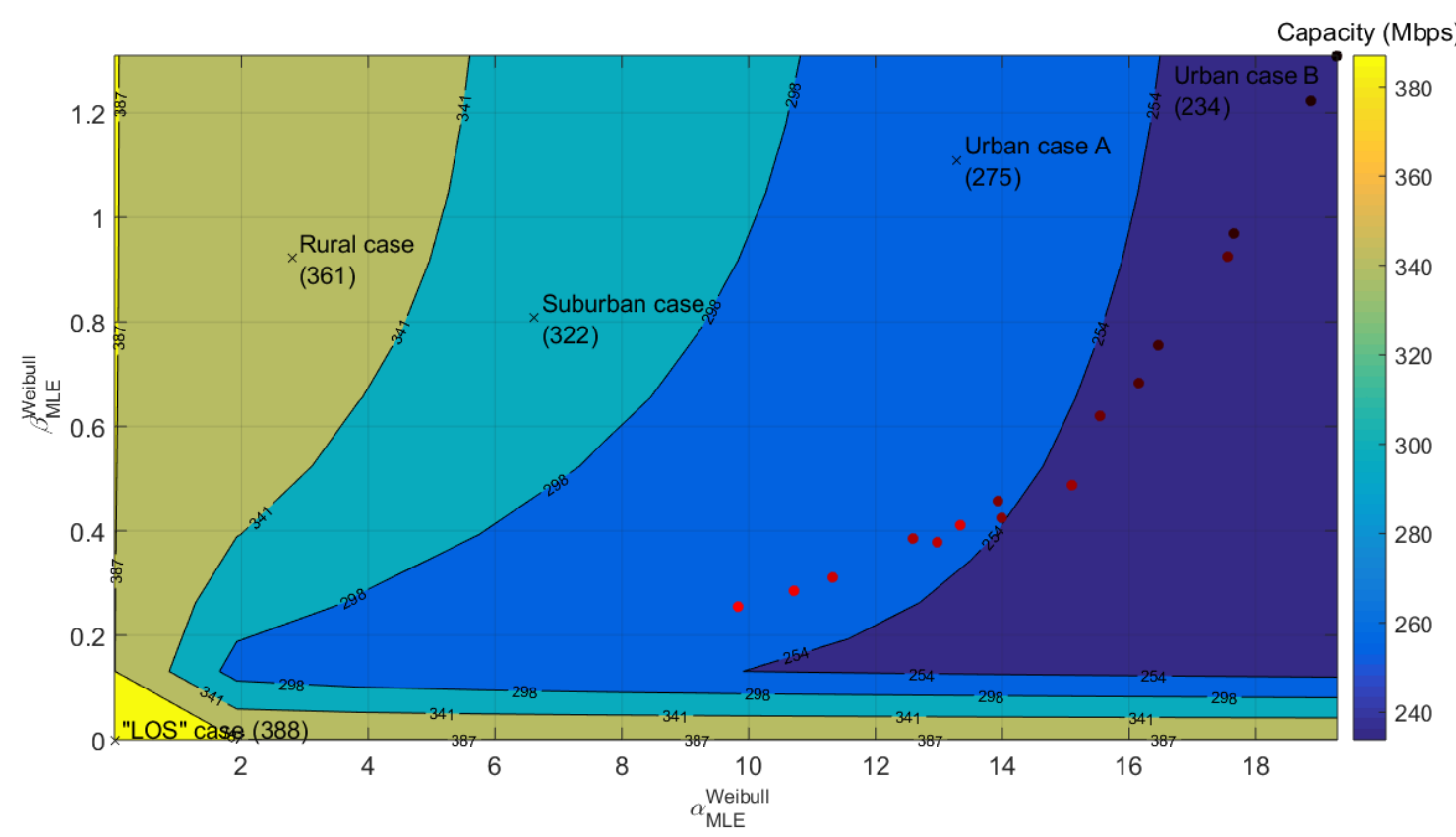

(b) 


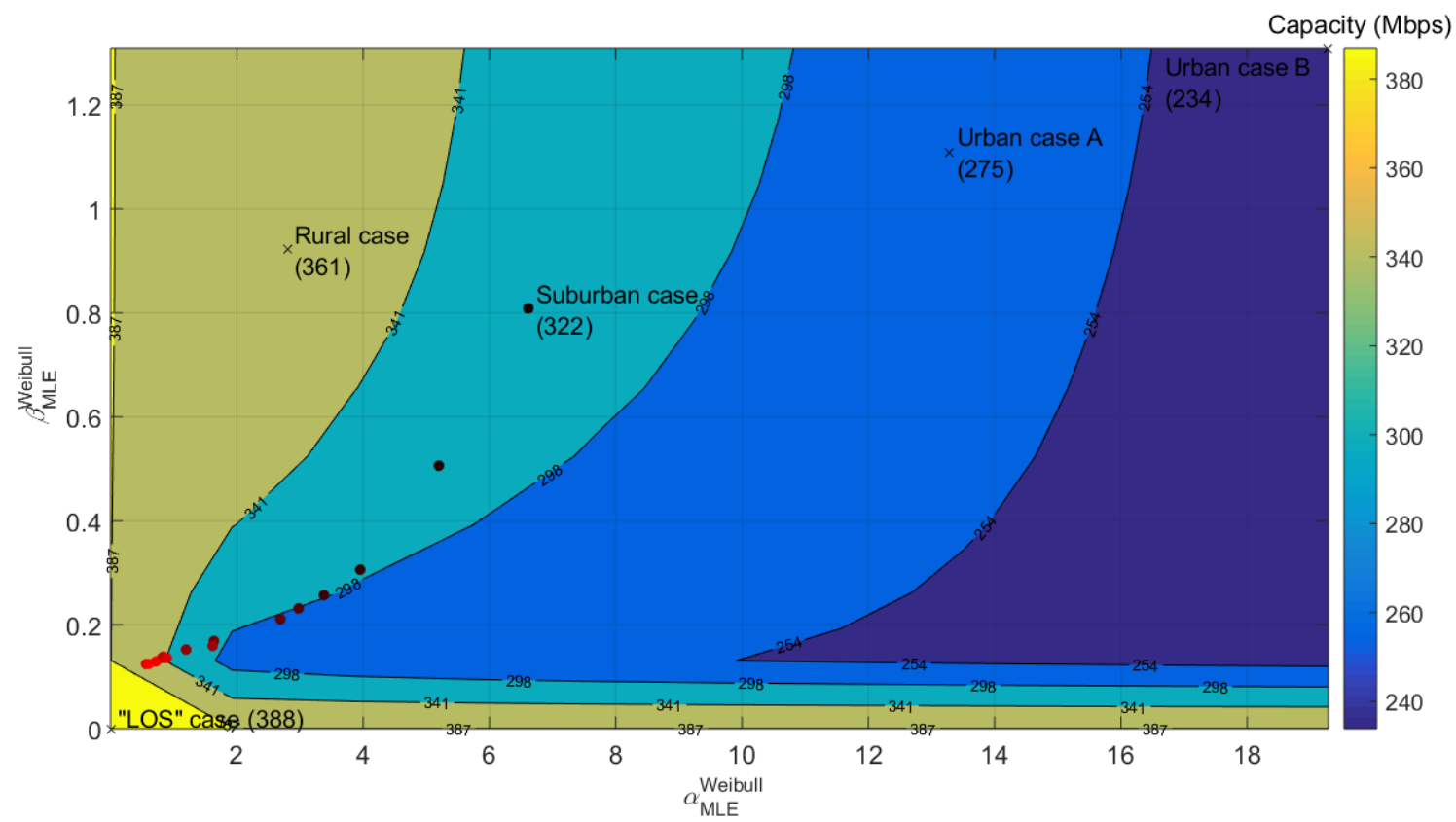

(c)

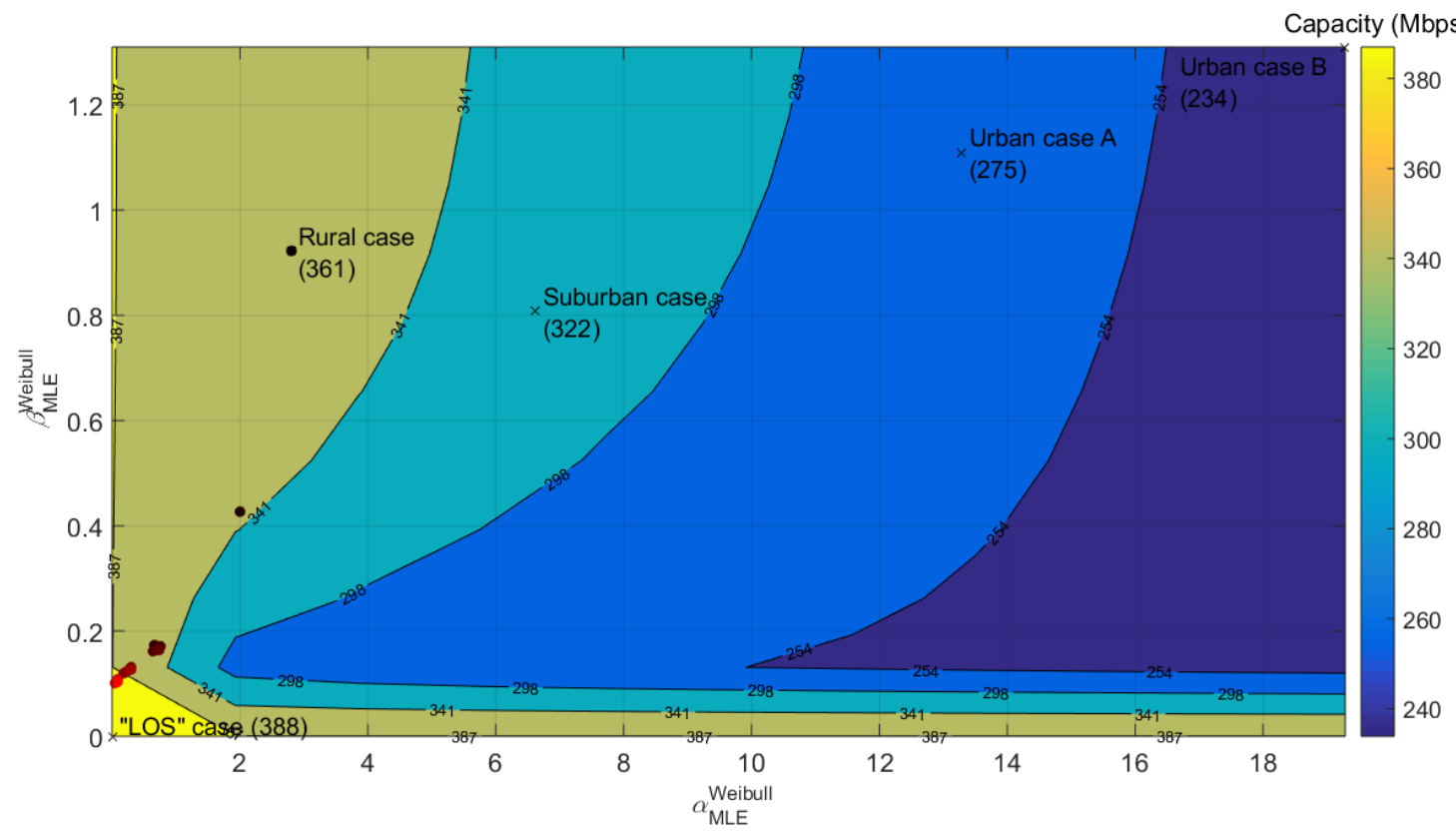

(d) 


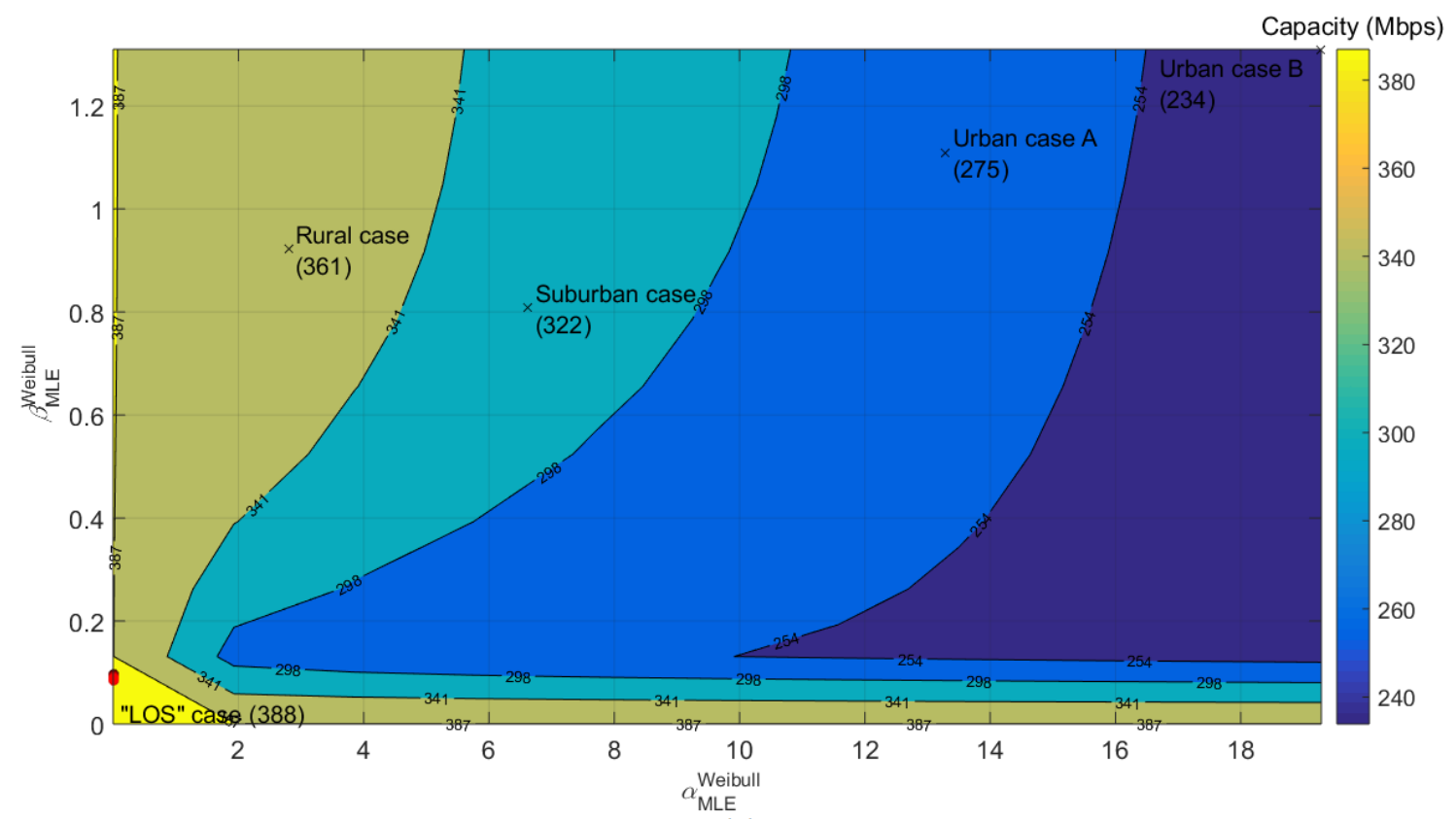

(e)

Fig. 4. iSHM footprints of the real indicative OV LV BPL topologies when 3-30MHz frequency band, $\mathrm{WtG}^{1}$ coupling scheme, FCC Part 15 and CUD measurement differences of maximum value $a_{\mathrm{CUD}}$ that ranges from $0 \mathrm{~dB}$ (black spot) to $15 \mathrm{~dB}$ (red spot) are assumed. (i) Urban case A. (ii) Urban case B (iii) Suburban case (iv) Rural case (v) "LOS" case. 


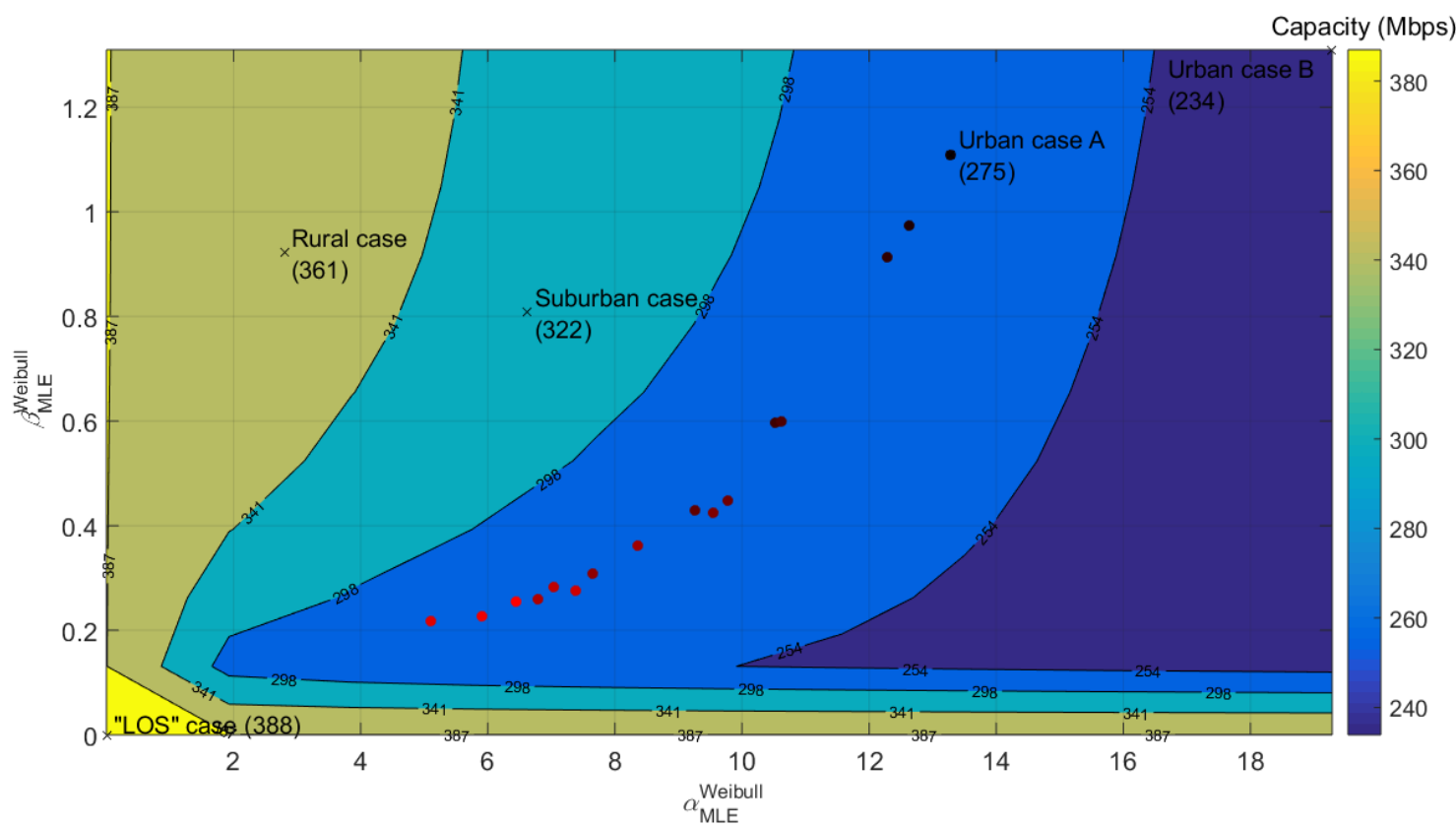

(a)

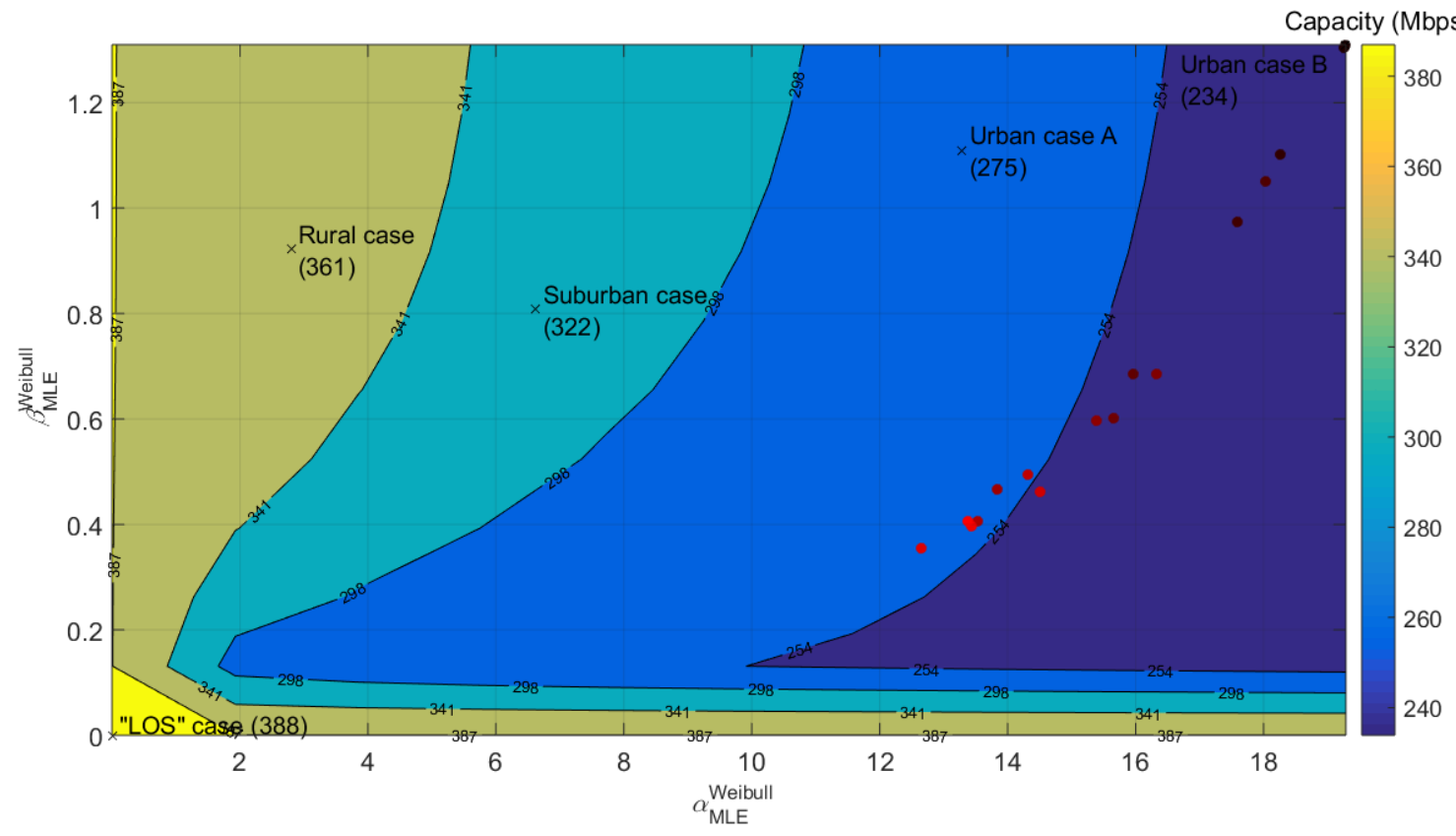

(b) 


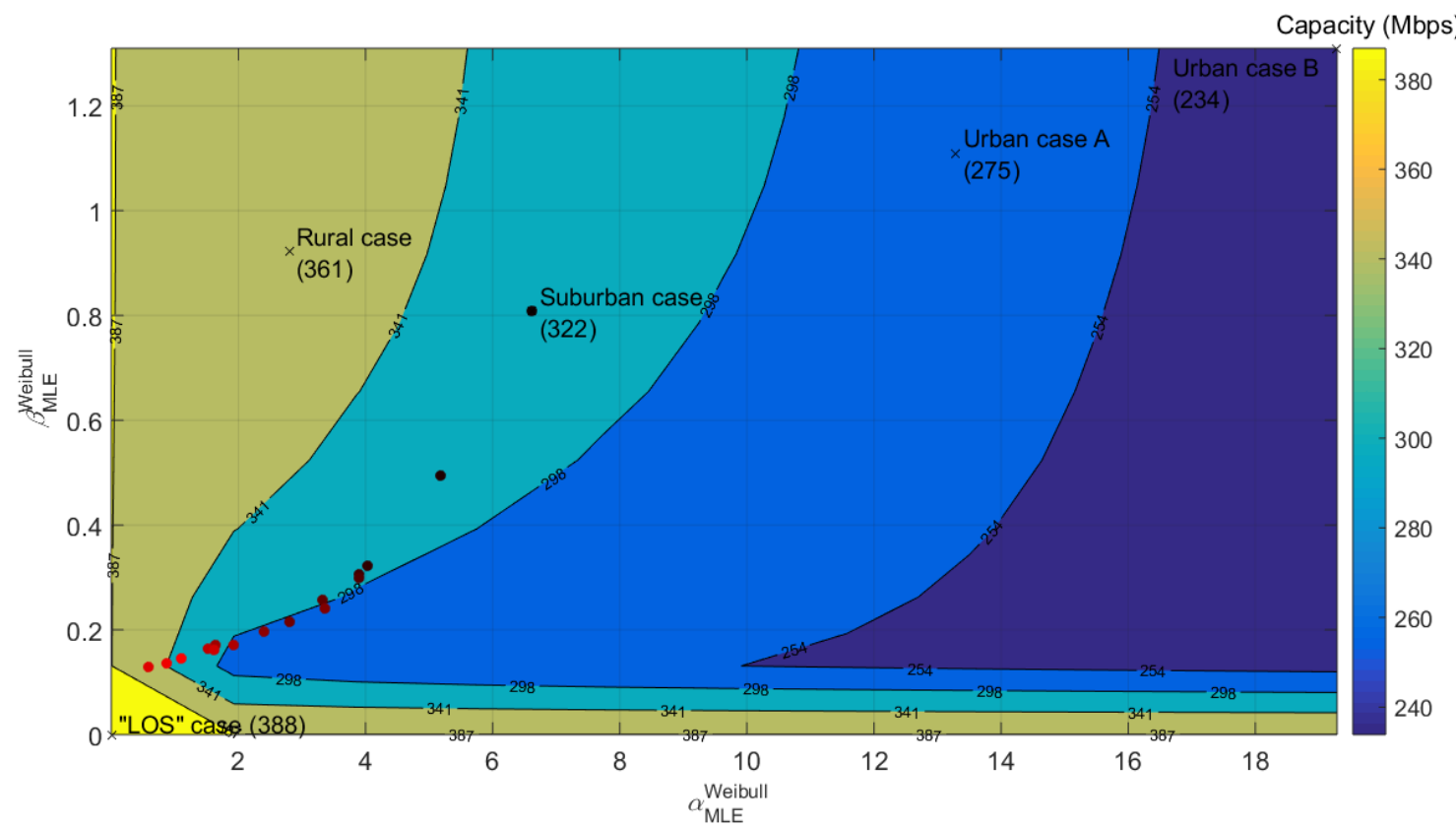

(c)

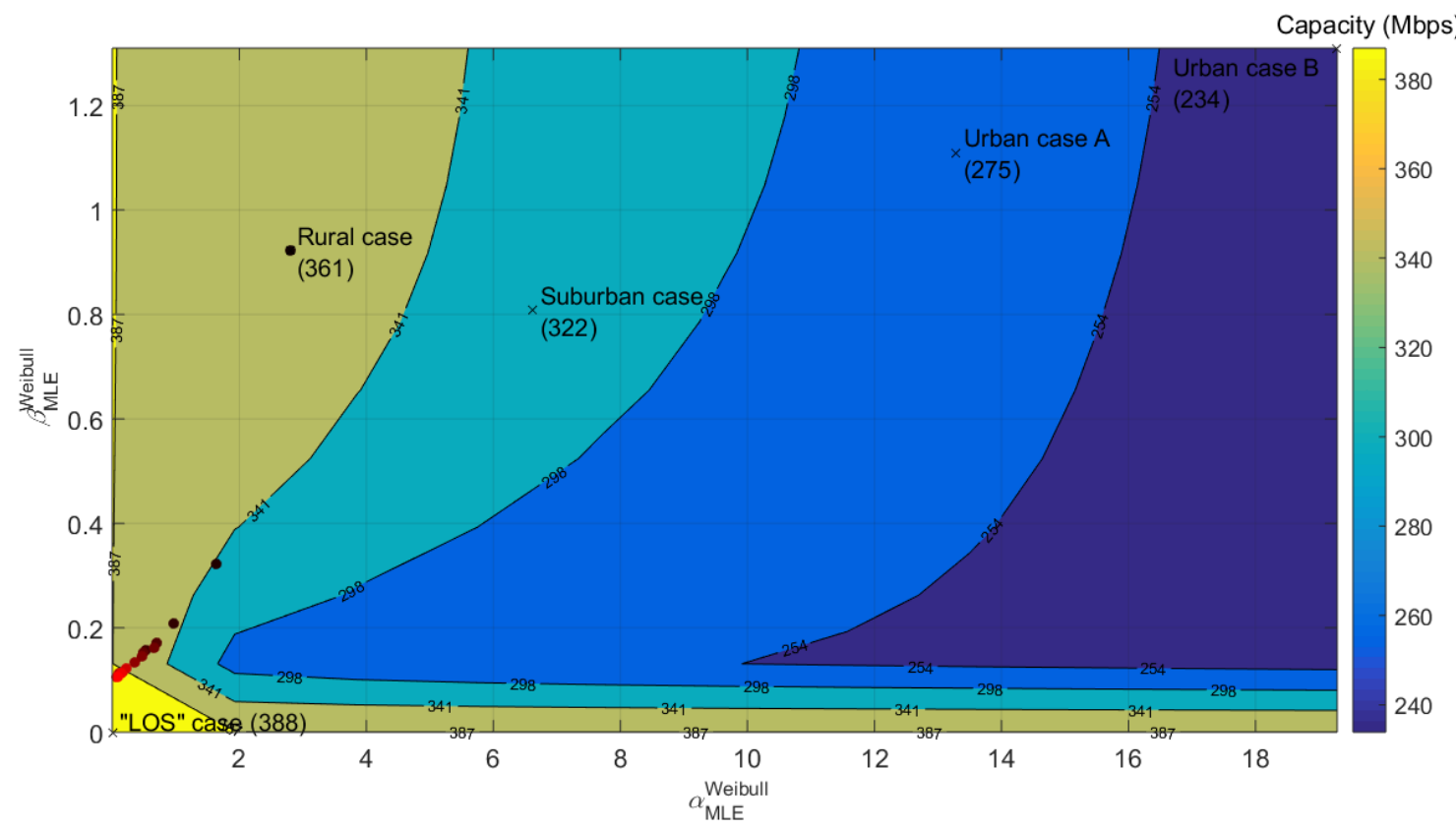

(d) 


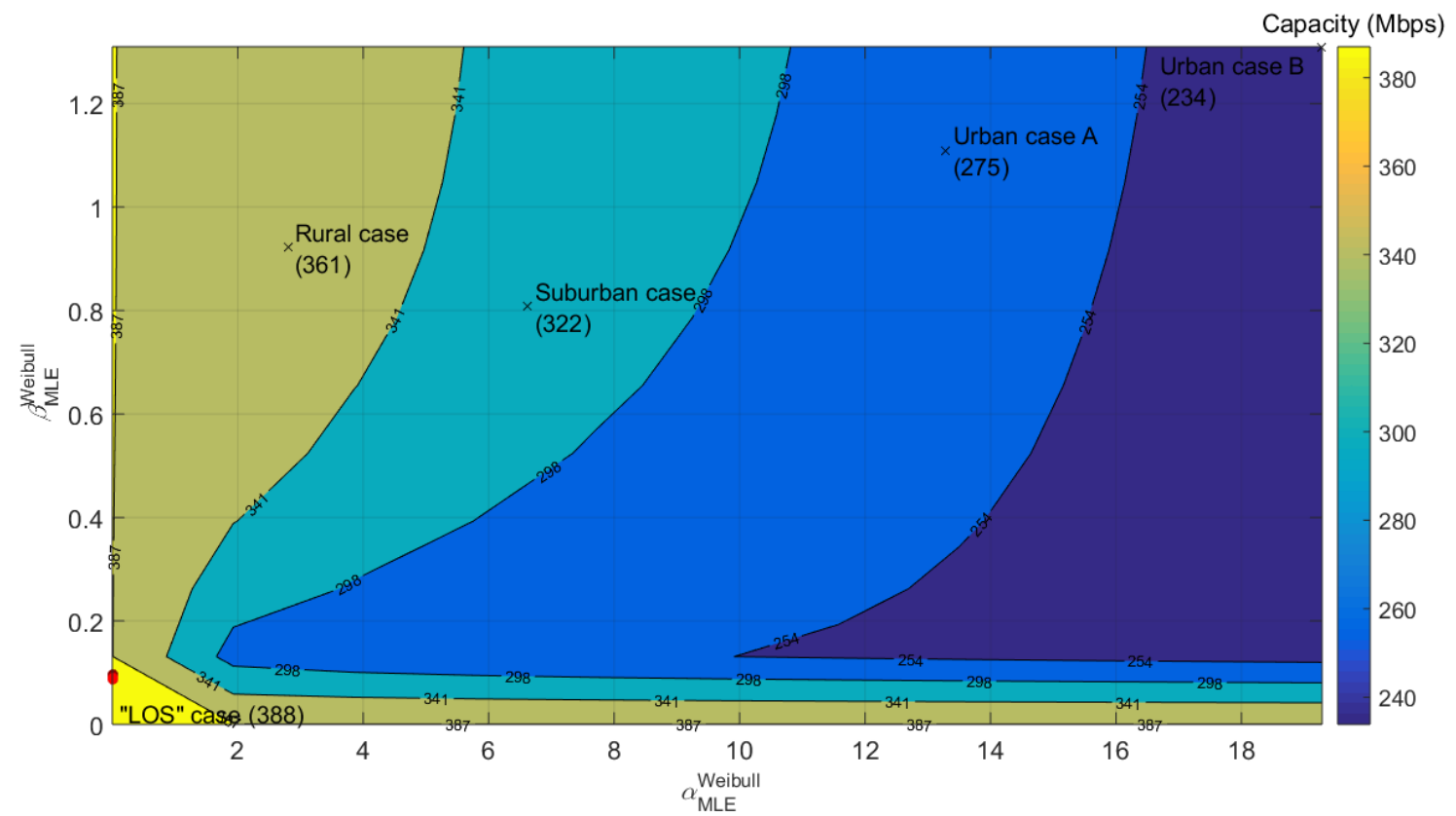

(e)

Fig. 5. Same plots but for ND measurement difference of mean $\mu_{\mathrm{ND}}=0 \mathrm{~dB}$ and standard deviation $\sigma_{\mathrm{ND}}$ that ranges from $0 \mathrm{~dB}$ (black spot) to $7.5 \mathrm{~dB}$ (red spot).

By combining Figs. 4(a)-(e) and 5(a)-(e) with the respective figures of the previous subsections, interesting conclusions can be deduced regarding the behavior of iSHM footprints when measurement differences of different intensities are applied. More specifically:

- As the maximum value $a_{\text {CUD }}$ of CUD measurement differences or standard deviation $\sigma_{\mathrm{ND}}$ of $\mathrm{ND}$ measurement differences increase, $\hat{a}_{\mathrm{MLE}}^{\mathrm{Weibull}}$ and $\hat{\beta}_{\mathrm{MLE}}^{\mathrm{Weibull}}$ of the measured real indicative OV LV BPL topologies simultaneously decrease. The iSHM footprint that is created from the increasing measurement differences, denoted hereafter as iSHM footprint due to the increasing measurement differences, starts from the theoretical values of $\hat{a}_{\mathrm{MLE}}^{\text {Weibull }}$ and $\hat{\beta} \mathrm{MLE}$ Weibull of the examined representative OV LV BPL topologies and tends to the iSHM footprint of "LOS" case that is located very close to the axes origin. The trend of the iSHM footprint due to the increasing measurement differences is explained by the fact that higher measurement differences force the measured coupling scheme channel attenuation of the real indicative OV LV BPL topologies to the zero restriction of the measured coupling scheme channel attenuation difference or to deeper spectral notches. Hence, the decrease of $\hat{a}_{\mathrm{MLE}}^{\mathrm{Weibull}}$ and $\hat{\beta}_{\mathrm{MLE}}^{\mathrm{Weibull}}$ of the measured real indicative OV LV BPL topologies compared with the respective theoretical ones is explained by the effort of the Weibull PDF to approximate the empirical PDFs of the measured coupling scheme channel attenuation differences.

- By observing Figs. 4(e) and 5(e), the iSHM footprint of the OV LV BPL "LOS" case due to the increasing measurement differences remains close to the axes origin. In fact, the distance of the iSHM footprint of the OV LV BPL "LOS" case from the axes origin when no measurement differences are applied is explained by 
the fact that the coupling scheme channel attenuation differences is assumed to be equal to $1 \times 10^{-11}$ instead of zero so that Weibull CASD MLEs can be calculated [19].

- The iSHM footprint due to the increasing measurement differences depends on the examined OV LV BPL topology class since its trend becomes steeper as more aggravated BPL topologies are studied. Actually, for high values of maximum value $a_{\mathrm{CUD}}$ of CUD measurement differences and standard deviation $\sigma_{\mathrm{ND}}$ of ND measurement differences, the iSHM footprint due to the increasing measurement differences can exceed the boundaries of the examined OV LV BPL topology class. As it was expected, the latter situation complicates either the identification of the examined OV LV BPL topology or the categorization of the examined OV LV BPL topology into the five classes.

- The significant Weibull MLEs distortion that is caused by the presence of high measurement differences may affect the monitoring and controlling of the OV LV power grid [28], [29]. To mitigate the measurement differences and preserve the validity of the collected data, piecewise monotonic data approximations, such as L1PMA [27] and L2WPMA [53], have been extensively applied in distribution and transmission BPL networks until now so that measured data can be filtered and restored. The future research is focused on the exploitation of the piecewise monotonic data approximations so that the lower left direction of the iSHM footprint due to measurement differences towards the axes origin can be inverted so that predictive tools that can exploit SHM footprints, such as the identification of OV LV BPL topologies and the detection of hooks for energy thefts [2], are not critically affected.

As been demonstrated, the operation of the SG produces big data whose wiser management may allow higher performances concerning the monitoring and controlling of the SG. Indeed, measurement differences combined with the real time operation are going to create a cataclysm of data that may mislead the existing predictive tools of the SG if they are not appropriately filtered. The interaction of iSHM footprints with piecewise monotonic data approximations may enhance the quality of business analytics of SG under the harsh real time conditions.

\section{Conclusions}

The numerical results concerning the behavior of iSHM footprints of the OV LV BPL networks when measurement differences are applied have been demonstrated as well as countermeasures proposals for ensuring the quality of business analytics and the tools of the SG. From iSHM footprints of the real indicative OV LV BPL topologies due to the increasing measurement differences, it has been verified that: (i) iSHM footprints of more aggravated OV LV BPL topologies (i.e., urban case A and case B) are more sensitive to the measurement differences (higher extent of the corresponding iSHM footprints); (ii) When measurement differences remain relatively low, the iSHM footprint of the affected OV LV BPL topology remains within the corresponding class area boundaries; (iii) iSHM footprints due to the increasing measurement differences of all examined real OV LV BPL topologies present the same direction; say, towards the axes origin; (iv) As the measurement differences remain high, all affected OV LV BPL topologies tend to present similar Weibull CASD MLEs; and (v) High measurement differences jam the broadband tools of the SG, such as the 
topology identification technique via iSHM footprint, thus influencing the quality of business analytics of the SG. To mitigate the measurement differences and retrieve the theoretical iSHM footprint from the measured one, piecewise monotonic data approximations, such as L1PMA and L2WPMA, are investigated in [54], [55].

\section{CONFLICTS OF INTEREST}

The author declares that there is no conflict of interests regarding the publication of this paper.

\section{References}

[1] A. G. Lazaropoulos, "Statistical Channel Modeling of Overhead Low Voltage Broadband over Power Lines (OV LV BPL) Networks - Part 1: The Theory of Class Map Footprints of Real OV LV BPL Topologies, Branch Line Faults and Hook-Style Energy Thefts," Trends in Renewable Energy, vol. 6, no. 1, pp. 61-87, Mar. $2020.2 \quad$ [Online]. Available: http://futureenergysp.com/index.php/tre/article/download/112/pdf

[2] A. G. Lazaropoulos, "Statistical Channel Modeling of Overhead Low Voltage Broadband over Power Lines (OV LV BPL) Networks - Part 2: The Numerical Results of Class Map Footprints of Real OV LV BPL Topologies, Branch Line Faults and Hook Style Energy Thefts," Trends in Renewable Energy, vol. 6, no. 1, pp. 88-109, $2020 . \quad$ Mar. AOnline]. Available: http://futureenergysp.com/index.php/tre/article/download/113/pdf

[3] G. López, J. Matanza, D. de la Vega, M. Castro, A. Arrinda, J. I. Moreno, and A. Sendin, "The Role of Power Line Communications in the Smart Grid Revisited: Applications, Challenges, and Research Initiatives," IEEE Access, vol. 7, pp. 117346-117368, 2019.

[4] A. A. Munshi and A-R M. Yasser, "Big Data Framework for Analytics in Smart Grids," Elsevier Electric Power Systems Research, vol. 151, pp. 369-380, 2017.

[5] H. Daki, A. El Hannani, A. Aqqal, A. Haidine, and A. Dahbi, "Big Data management in smart grid: concepts, requirements and implementation," Springer Journal of Big Data, vol. 4, no. 1, pp. 1-19, 2017.

[6] A. H. Al-Badi, R. Ahshan, N. Hosseinzadeh, R. Ghorbani, and E. Hossain, "Survey of Smart Grid Concepts and Technological Demonstrations Worldwide Emphasizing on the Oman Perspective," MDPI Applied System Innovation, vol. 3, no. 1, 5, 2020.

[7] G. Artale, A. Cataliotti, V. Cosentino, D. Di Cara, R. Fiorelli, S. Guaiana, and G. Tinè, "A New Low Cost Coupling System for Power Line Communication on Medium Voltage Smart Grids," IEEE Trans. on Smart Grid, vol. 9, no. 4, pp. 3321-3329, 2018.

[8] L. González-Sotres, C. Mateo, P. Frías, C. Rodríguez-Morcillo, and J. Matanza, "Replicability Analysis of PLC PRIME Networks for Smart Metering Applications," IEEE Trans. on Smart Grid, vol. 9, no. 2, pp. 827-835, Mar. 2018. 
[9] A. ElSamadouny, A. El Shafie, M. Abdallah, and N. Al-Dhahir, "Secure SumRate-Optimal MIMO Multicasting Over Medium-Voltage NB-PLC Networks," IEEE Trans. on Smart Grid, vol. 9, no. 4, pp. 2954-2963, Jul. 2018.

[10] A. G. Lazaropoulos and P. G. Cottis, "Transmission characteristics of overhead medium voltage power line communication channels," IEEE Trans. Power Del., vol. 24, no. 3, pp. 1164-1173, Jul. 2009.

[11] A. G. Lazaropoulos and P. G. Cottis, "Broadband transmission via underground medium-voltage power lines-Part I: transmission characteristics," IEEE Trans. Power Del., vol. 25, no. 4, pp. 2414-2424, Oct. 2010.

[12] E. Biglieri, "Coding and modulation for a horrible channel," IEEE Commun. Mag., vol. 41, no. 5, pp. 92-98, May 2003.

[13] M. Gebhardt, F. Weinmann, and K. Dostert, "Physical and regulatory constraints for communication over the power supply grid," IEEE Commun. Mag., vol. 41, no. 5, pp. 84-90, May 2003.

[14] M. Götz, M. Rapp, and K. Dostert, "Power line channel characteristics and their effect on communication system design," IEEE Commun. Mag., vol. 42, no. 4, pp. 78-86, Apr. 2004.

[15] A. G. Lazaropoulos and P. G. Cottis, "Capacity of overhead medium voltage power line communication channels," IEEE Trans. Power Del., vol. 25, no. 2, pp. 723-733, Apr. 2010.

[16] A. G. Lazaropoulos and P. G. Cottis, "Broadband transmission via underground medium-voltage power lines-Part II: capacity," IEEE Trans. Power Del., vol. 25, no. 4, pp. 2425-2434, Oct. 2010.

[17] A. G. Lazaropoulos, "Broadband transmission and statistical performance properties of overhead high-voltage transmission networks," Hindawi Journal of Computer Networks and Commun., 2012, article ID 875632, 2012. [Online]. Available: http://www.hindawi.com/journals/jcnc/aip/875632/

[18] A. G. Lazaropoulos, "Towards Modal Integration of Overhead and Underground Low-Voltage and Medium-Voltage Power Line Communication Channels in the Smart Grid Landscape: Model Expansion, Broadband Signal Transmission Characteristics, and Statistical Performance Metrics (Invited Paper)," ISRN Signal Processing, vol. 2012, Article ID 121628, pp. 1-17, 2012. [Online]. Available: http://www.hindawi.com/isrn/sp/2012/121628/

[19] A. G. Lazaropoulos, "Statistical Broadband over Power Lines Channel Modeling - Part 1: The Theory of the Statistical Hybrid Model," Progress in Electromagnetics Research C, vol. 92, pp. 1-16, 2019. [Online]. Available: http://www.jpier.org/PIERC/pierc92/01.19012902.pdf

[20] A. G. Lazaropoulos, "Statistical Broadband over Power Lines (BPL) Channel Modeling - Part 2: The Numerical Results of the Statistical Hybrid Model," Progress in Electromagnetics Research C, vol. 92, pp. 17-30, 2019. [Online]. Available: http://www.jpier.org/PIERC/pierc92/02.19012903.pdf

[21] A. G. Lazaropoulos, "Enhancing the Statistical Hybrid Model Performance in Overhead and Underground Medium Voltage Broadband over Power Lines Channels by Adopting Empirical Channel Attenuation Statistical Distribution," Trends in Renewable Energy, vol. 5, no. 2, pp. 181-217, 2019. [Online]. Available: http://futureenergysp.com/index.php/tre/article/view/96/pdf

[22] A. G. Lazaropoulos, "The Role of Information Technology Department against the Hook Style Energy Theft in Smart Cities - Ad-Hoc Overhead Low-Voltage 
Broadband over Power Lines (OV LV BPL) Networks," Trends in Renewable Energy, vol. 5, no. 2, pp. 117-150, Apr. 2019. [Online]. Available: http://futureenergysp.com/index.php/tre/article/download/93/pdf

[23] A. G. Lazaropoulos, "Virtual Indicative Broadband over Power Lines Topologies for Respective Subclasses by Adjusting Channel Attenuation Statistical Distribution Parameters of Statistical Hybrid Models - Part 2: Numerical Results for the Overhead and Underground Medium-Voltage Power Grids," Trends in Renewable Energy, vol. 5, no. 3, pp. 258-281, Aug. 2019. [Online]. Available: http://futureenergysp.com/index.php/tre/article/view/100/pdf

[24] A. G. Lazaropoulos, "Virtual Indicative Broadband over Power Lines Topologies for Respective Subclasses by Adjusting Channel Attenuation Statistical Distribution Parameters of Statistical Hybrid Models - Part 3: The Case of Overhead Transmission Power Grids," Trends in Renewable Energy, vol. 5, no. 3, pp. 282-306, Aug. $2019 . \quad$ [Online]. Available: http://futureenergysp.com/index.php/tre/article/view/101/pdf

[25] A. G. Lazaropoulos, "Measurement Differences, Faults and Instabilities in Intelligent Energy Systems-Part 1: Identification of Overhead High-Voltage Broadband over Power Lines Network Topologies by Applying Topology Identification Methodology (TIM)," Trends in Renewable Energy, vol. 2, no. 3, pp. 85-112, Oct. 2016. [Online]. Available: http://futureenergysp.com/index.php/tre/article/view/26/32

[26] A. G. Lazaropoulos, "Measurement Differences, Faults and Instabilities in Intelligent Energy Systems - Part 2: Fault and Instability Prediction in Overhead High-Voltage Broadband over Power Lines Networks by Applying Fault and Instability Identification Methodology (FIIM)," Trends in Renewable Energy, vol. 2, no. 3, pp. 113-142, Oct. 2016. [Online]. Available: http://futureenergysp.com/index.php/tre/article/view/27/33

[27] A. G. Lazaropoulos, "Best L1 Piecewise Monotonic Data Approximation in Overhead and Underground Medium-Voltage and Low-Voltage Broadband over Power Lines Networks: Theoretical and Practical Transfer Function Determination," Hindawi Journal of Computational Engineering, vol. 2016, Article ID 6762390, 24 pages, 2016. doi:10.1155/2016/6762390. [Online]. Available: https://www.hindawi.com/journals/jcengi/2016/6762390/cta/

[28] A. G. Lazaropoulos, "Power Systems Stability through Piecewise Monotonic Data Approximations - Part 1: Comparative Benchmarking of L1PMA, L2WPMA and L2CXCV in Overhead Medium-Voltage Broadband over Power Lines Networks," Trends in Renewable Energy, vol. 3, no. 1, pp. 2-32, Jan. 2017. [Online]. Available: http://futureenergysp.com/index.php/tre/article/view/29/34

[29] A. G. Lazaropoulos, "Power Systems Stability through Piecewise Monotonic Data Approximations - Part 2: Adaptive Number of Monotonic Sections and Performance of L1PMA, L2WPMA and L2CXCV in Overhead Medium-Voltage Broadband over Power Lines Networks," Trends in Renewable Energy, vol. 3, no. 1, pp. 33-60, Jan. 2017. [Online]. Available: http://futureenergysp.com/index.php/tre/article/view/30/35

[30] A. G. Lazaropoulos, "Main Line Fault Localization Methodology in Smart Grid Part 2: Extended TM2 Method, Measurement Differences and L1 Piecewise Monotonic Data Approximation for the Overhead Medium-Voltage Broadband over Power Lines Networks Case," Trends in Renewable Energy, vol. 3, no. 3, pp. 
26-61, Dec. 2017. [Online]. Available: http://futureenergysp.com/index.php/tre/article/view/37

[31] OPERA1, D44: Report presenting the architecture of plc system, the electricity network topologies, the operating modes and the equipment over which PLC access system will be installed, IST Integr. Project No 507667, Dec. 2005.

[32] P. Amirshahi and M. Kavehrad, "High-frequency characteristics of overhead multiconductor power lines for broadband communications," IEEE J. Sel. Areas Commun., vol. 24, no. 7, pp. 1292-1303, Jul. 2006.

[33] P. Amirshahi, "Broadband access and home networking through powerline networks” Ph.D. dissertation, Pennsylvania State Univ., University Park, PA, May 2006.

[34] M. D'Amore and M. S. Sarto, "A new formulation of lossy ground return parameters for transient analysis of multiconductor dissipative lines," IEEE Trans. Power Del., vol. 12, no. 1, pp. 303-314, Jan. 1997.

[35] M. D'Amore and M. S. Sarto, "Simulation models of a dissipative transmission line above a lossy ground for a wide-frequency range-Part I: Single conductor configuration," IEEE Trans. Electromagn. Compat., vol. 38, no. 2, pp. 127-138, May 1996.

[36] M. D'Amore and M. S. Sarto, "Simulation models of a dissipative transmission line above a lossy ground for a wide-frequency range-Part II: Multi-conductor configuration," IEEE Trans. Electromagn. Compat., vol. 38, no. 2, pp. 139-149, May 1996.

[37] A. G. Lazaropoulos, "Review and Progress towards the Capacity Boost of Overhead and Underground Medium-Voltage and Low-Voltage Broadband over Power Lines Networks: Cooperative Communications through Two- and ThreeHop Repeater Systems," ISRN Electronics, vol. 2013, Article ID 472190, pp. 119, 2013. [Online]. Available: http://www.hindawi.com/isrn/electronics/aip/472190/

[38] A. G. Lazaropoulos, "Broadband over Power Lines (BPL) Systems Convergence: Multiple-Input Multiple-Output (MIMO) Communications Analysis of Overhead and Underground Low-Voltage and Medium-Voltage BPL Networks (Invited Paper)," ISRN Power Engineering, vol. 2013, Article ID 517940, pp. 1-30, 2013. [Online].

Available: http://www.hindawi.com/isrn/power.engineering/2013/517940/

[39] A. G. Lazaropoulos, "Main Line Fault Localization Methodology (MLFLM) in Smart Grid-The Underground Medium- and Low-Voltage Broadband over Power Lines Networks Case," Trends in Renewable Energy, vol. 4, no. 1, pp. 15-42, Dec. $2017 . \quad$ [Online]. Available: http://futureenergysp.com/index.php/tre/article/view/45

[40] A. G. Lazaropoulos, "Towards Broadband over Power Lines Systems Integration: Transmission Characteristics of Underground Low-Voltage Distribution Power Lines," Progress in Electromagnetics Research B, vol. 39, pp. 89-114, 2012. [Online]. Available: http://www.jpier.org/PIERB/pierb39/05.12012409.pdf

[41] T. Sartenaer, "Multiuser communications over frequency selective wired channels and applications to the powerline access network" Ph.D. dissertation, Univ. Catholique Louvain, Louvain-la-Neuve, Belgium, Sep. 2004.

[42] T. Calliacoudas and F. Issa, "“'Multiconductor transmission lines and cables solver," An efficient simulation tool for plc channel networks development," 
presented at the IEEE Int. Conf. Power Line Communications and Its Applications, Athens, Greece, Mar. 2002.

[43] T. Sartenaer and P. Delogne, "Deterministic modelling of the (Shielded) outdoor powerline channel based on the multiconductor transmission line equations," IEEE J. Sel. Areas Commun., vol. 24, no. 7, pp. 1277-1291, Jul. 2006.

[44] A. G. Lazaropoulos, "Broadband Performance Metrics and Regression Approximations of the New Coupling Schemes for Distribution Broadband over Power Lines (BPL) Networks," Trends in Renewable Energy, vol. 4, no. 1, pp. 43-73, Jan. $2018 . \quad$ [Online]. Available: http://futureenergysp.com/index.php/tre/article/view/59/pdf

[45] A. G. Lazaropoulos, "New Coupling Schemes for Distribution Broadband over Power Lines (BPL) Networks," Progress in Electromagnetics Research B, vol. 71, pp. 39-54, 2016. [Online]. Available: http://www.jpier.org/PIERB/pierb71/02.16081503.pdf

[46] A. G. Lazaropoulos, "Underground Distribution BPL Connections with $(\mathrm{N}+1)-$ hop Repeater Systems: A Novel Capacity Mitigation Technique," Elsevier Computers and Electrical Engineering, vol. 40, pp. 1813-1826, 2014.

[47] A. G. Lazaropoulos, "Deployment Concepts for Overhead High Voltage Broadband over Power Lines Connections with Two-Hop Repeater System: Capacity Countermeasures against Aggravated Topologies and High Noise Environments," Progress in Electromagnetics Research B, vol. 44, pp. 283-307, 2012. [Online]. Available: http://www.jpier.org/PIERB/pierb44/13.12081104.pdf

[48] A. G. Lazaropoulos, "Virtual Indicative Broadband over Power Lines Topologies for Respective Subclasses by Adjusting Channel Attenuation Statistical Distribution Parameters of Statistical Hybrid Models - Part 1: Theory," Trends in Renewable Energy, vol. 5, no. 3, pp. 237-257, Aug. 2019. [Online]. Available: http://futureenergysp.com/index.php/tre/article/view/99/pdf

[49] A. G. Lazaropoulos, "Improvement of Power Systems Stability by Applying Topology Identification Methodology (TIM) and Fault and Instability Identification Methodology (FIIM)-Study of the Overhead Medium-Voltage Broadband over Power Lines (OV MV BPL) Networks Case," Trends in Renewable Energy, vol. 3, no. 2, pp. 102-128, Apr. 2017. [Online]. Available: http://futureenergysp.com/index.php/tre/article/view/34

[50] I. C. Demetriou, "An application of best $L 1$ piecewise monotonic data approximation to signal restoration," IAENG International Journal of Applied Mathematics, vol. 53, no. 4, pp. 226-232, 2013.

[51] I. C. Demetriou, "L1PMA: A Fortran 77 Package for Best L1 Piecewise Monotonic Data Smoothing," Computer Physics Communications, vol. 151, no. 1, pp. 315-338, 2003.

[52] A. G. Lazaropoulos, "Main Line Fault Localization Methodology in Smart Grid Part 1: Extended TM2 Method for the Overhead Medium-Voltage Broadband over Power Lines Networks Case," Trends in Renewable Energy, vol. 3, no. 3, pp. 2-25, Dec. 2017. [Online]. Available: http://futureenergysp.com/index.php/tre/article/view/36

[53] I. C. Demetriou, "Algorithm 863: L2WPMA, a Fortran 77 package for weighted least-squares piecewise monotonic data approximation," ACM Transactions on Mathematical Software (TOMS), vol. 33, no.1, pp. 6, 2007. 
[54] A. G. Lazaropoulos, "Business Analytics and IT in Smart Grid - Part 2: The Qualitative Mitigation Impact of Piecewise Monotonic Data Approximations on the iSHM Class Map Footprints of Overhead Low-Voltage Broadband over Power Lines Topologies Contaminated by Measurement Differences," Trends in Renewable Energy, vol. 6, no. 2, pp. 177-203, May 2020.

[55] A. G. Lazaropoulos, "Business Analytics and IT in Smart Grid - Part 3: New Application Aspect and the Quantitative Mitigation Analysis of Piecewise Monotonic Data Approximations on the iSHM Class Map Footprints of Overhead Low-Voltage Broadband over Power Lines Topologies Contaminated by Measurement Differences," Trends in Renewable Energy, vol. 6, no. 2, pp. 204223, May 2020.

Article copyright: (C) 2020 Athanasios G. Lazaropoulos. This is an open access article distributed under the terms of the Creative Commons Attribution 4.0 International License, which permits unrestricted use and distribution provided the original author and source are credited. 